\title{
Collider limits on leptophilic interactions
}

\author{
Francisco del Aguila, ${ }^{a}$ Mikael Chala, ${ }^{a, b}$ Jose Santiago ${ }^{a, c}$ and Yasuhiro Yamamoto ${ }^{a}$ \\ ${ }^{a}$ CAFPE and Departamento de Física Teórica y del Cosmos, Universidad de Granada, \\ E-18071 Granada, Spain \\ ${ }^{b} D E S Y$, \\ Notkestrasse 85, 22607 Hamburg, Germany \\ ${ }^{c}$ CERN, Theory Division, \\ CH-1211 Geneva 23, Switzerland \\ E-mail: faguila@ugr.es, mikael.chala@desy.de, jsantiago@ugr.es, \\ yamayasu@ugr.es
}

ABSTRACT: Leptophilic interactions can only be observed at the LHC in four-lepton final states. If these interactions are mediated by a resonance in the di-leptonic channel with renormalizable couplings, the mediator must have spin 1 . We study the LHC reach for such a vector boson allowing for arbitrary couplings. We find that only couplings to muons can be probed at the LHC because lepton flavor violating couplings are constrained by rare processes, couplings to electrons by LEP and the LHC is not sensitive to final states involving taus in this case. The ILC becomes then complementary to the LHC as it will provide the best limits on $Z^{\prime}$ couplings to tau leptons. A prominent example is the case of the anomaly-free $Z^{\prime}$ coupling to the muon minus tau lepton number $\mathrm{L}_{\mu}-\mathrm{L}_{\tau}$. If no departure from the Standard Model is observed at the LHC, the most stringent bounds on this vector boson are provided from events with only three charged leptons plus missing energy. Masses of the order of $1 \mathrm{TeV}$ can be probed at the high-luminosity phase of the LHC for $Z^{\prime}$ couplings of order one. Generic four-lepton operators parametrizing leptophilic interactions can be also constrained using three and four (or two at the ILC) charged-lepton samples, but the corresponding limits are marginal, if meaningful, because the resonant behavior appears to be essential for the signal to be significant.

Keywords: Beyond Standard Model, Technicolor and Composite Models

ARXiv EPRINT: 1411.7394 


\section{Contents}

1 Introduction 1

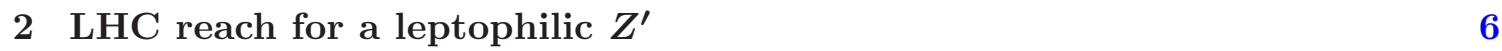

$\begin{array}{lll}3 & \text { ILC reach for a leptophilic } Z^{\prime} & 13\end{array}$

$\begin{array}{lll}4 & \text { Large collider bounds on four-lepton invariant operators } & 17\end{array}$

5 Conclusions $\quad 20$

\section{Introduction}

The Large Hadron Collider (LHC) is the largest discovery machine ever built. So far only the last particle within the Standard Model (SM), the Brout-Englert-Higgs (BEH) boson $[1,2]$, has been observed for the first time [3, 4]. Nevertheless, the outstanding performance of the LHC Collaborations has allowed to set stringent limits on many different SM extensions, especially on strongly interacting particles coupling directly to the initial partons. For instance, present bounds on dijet resonances are in the $3-5 \mathrm{TeV}$ region $[5,6]$, bounds on quark compositeness are of the order of $2.5 \mathrm{TeV}$ [7] and bounds on new quarks around $800 \mathrm{GeV}$ [8-10], respectively. ${ }^{1}$ Similar bounds, however, apply to resonances coupling with Electro-Weak (EW) strength to quarks and leptons due to the smaller SM backgrounds when the final state is not purely hadronic and includes isolated leptons with large transverse momentum. For instance, present direct bounds for a new $Z^{\prime}$ contributing to Drell-Yan production, $q \bar{q} \rightarrow Z^{\prime} \rightarrow l^{+} l^{-}$, can be up to $3 \mathrm{TeV}$ for popular SM additions $[14,15]$, as no departure from the SM prediction has been observed yet. Obviously, a hadron machine with an excellent lepton reconstruction is the proper place to look for New Physics (NP) characterized by resonances coupling sizably to both, quarks and leptons, or by the corresponding four-fermion effective operators if these new particles are banished to higher energies [16-18] (see also [19]). If so, little room will be left by the LHC for the discovery of new mediators of the reverse process, $l^{+} l^{-} \rightarrow q \bar{q}$, at future lepton colliders, if their Center of Mass Energy (CME) is moderate, as in the case of the International Linear Collider (ILC) (see [20] and references there in).$^{2}$

\footnotetext{
${ }^{1}$ Bounds on gluinos and squarks involve more partons in the final state and are more model dependent, being of the order of $1 \mathrm{TeV}$ or near this value, repectively [11-13].

${ }^{2}$ The ILC is foreseen as a precision machine and although its CME will not allow to produce relatively heavy particles, it will be able to provide indications or indirect constraints on NP well above its production threshold. A prime example is the case of an extra $Z^{\prime}$, for which the diagnostic reach at the ILC can be in general larger than at the LHC [21, 22]. However, although the discriminating power between different $Z^{\prime}$ additions can be better at the ILC, especially if polarized beams are available and for a higher CME,
} 
In this paper we want to address an alternative question: what can we learn at large (hadron) colliders about NP which couples only to leptons? Or in other words, which are the prospects to discover or to exclude leptophilic interactions at the LHC and/or ILC? The departures from the SM predictions in such a case have to be small, at least at the LHC, because the new processes must involve final leptons to which to attach the leptophilic interaction and they are produced with EW strength (in the SM) at a hadronic machine. In order to enhance the signal significance we may also require that it resonates in the di-lepton channel. This defines the most favorable scenario and we shall discuss it first.

Such a leptophilic particle must be a boson, for it couples to two fermions. Moreover, assuming renormalizable couplings, the new particle must be a vector boson if the SM gauge symmetry and matter content fully describe physics below the EW scale, as the LHC data seem to indicate. Indeed, a scalar multiplet transforming trivially under Lepton Number (LN) transformations can only couple to two SM lepton multiplets in a renormalizable way as does the SM scalar doublet but then, it also couples to the EW gauge bosons, and not only to leptons, as we assume. If we obviate this possibility by requiring the scalar to be a neutral singlet, we can only couple it to lepton pairs through non-renormalizable terms, which are in general effectively suppressed by small lepton masses because the corresponding lepton product must involve two multiplets with the same (wrong) chirality or an extra BEH boson insertion. Finally, if the new scalar multiplet has non-zero LN (in fact, equal to 2), it must have a doubly-charged component, coupling again to the SM gauge bosons, too (see refs. [24-27] for a detailed discussion of the lowest order couplings and production mechanisms of these extra scalars). In summary, the only leptophilic particle with renormalizable couplings to SM lepton pairs is a new neutral vector boson $Z^{\prime 3}$ with the following interaction Lagrangian:

$$
\mathcal{L}_{Z^{\prime}}=-\left(g_{\mathrm{L}}^{i j} \bar{L}_{\mathrm{L} i} \gamma^{\mu} L_{\mathrm{L} j}+g_{\mathrm{R}}^{\prime i j} \bar{l}_{\mathrm{R} i} \gamma^{\mu} l_{\mathrm{R} j}\right) Z_{\mu}^{\prime},
$$

where $g_{\mathrm{L}, \mathrm{R}}^{\prime i j}$ are arbitrary dimensionless couplings to the SM Left-Handed (LH) and RightHanded (RH) lepton multiplets, $L_{\mathrm{L} i}=\left(\begin{array}{c}\nu_{\mathrm{L} i} \\ l_{\mathrm{L} i}\end{array}\right)$ and $l_{\mathrm{R} i}$, respectively, with $i=e, \mu, \tau$ labeling the charged-lepton family. ${ }^{4}$ Higher-spin particles do not have renormalizable couplings to lepton pairs either.

it will be a challenging task to establish the existence of a new vector boson coupling to both quarks and leptons at this machine if no signal is observed at the LHC with a high luminosity. See [23] for a review and further references.

${ }^{3}$ Obviously, such a vector boson can mix with the $\mathrm{Z}$ boson $[28,29]$ and hence, also contribute to DrellYan production. As a matter of fact, this mixing is generated by quantum corrections in models with generic couplings if the mixing term is not already present after integrating out the heavy modes of a more fundamental theory at higher energies [30-32]. Such a mixing is in general small, as already experimentally required by present LHC bounds on new vector bosons contributing to Drell-Yan production [14, 15] (see also [33], and references there in). These limits then make negligible its contribution to four-fermion $Z^{\prime}$ final states and hence, we can neglect this mixing throughout the paper when studying the leading contribution to four-lepton production mediated by a leptophilic vector boson. (See ref. [34] for a more detailed discussion.)

${ }^{4}$ Thus, although arbitrary flavor and chiral interactions are allowed, the EW gauge symmetry remains unbroken for the new couplings of the LH charged leptons and their neutrino counterparts are equal. However, the SM Yukawa couplings do not preserve such a hypothetical gauge symmetry. As a matter of fact, 


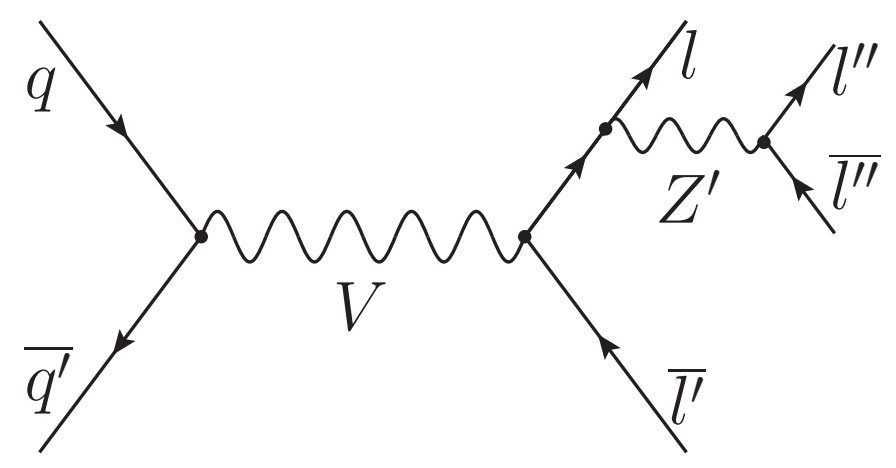

Figure 1. Leading production diagram of a leptophilic $Z^{\prime}$ at the LHC.

In the following we shall discuss the phenomenology of such a vector boson, leaving to the end the comments on the case where it is beyond the LHC reach and its effects are parametrized by the corresponding four-lepton operators at low energy. There are different NP sources for four-lepton signals at large colliders, as, for instance, new doubly-charged scalars introduced above or heavy neutrinos decaying into three leptons, with also different production mechanisms. Thus, whereas in the former case the dominant contribution results from EW pair production [24-27], in the latter it is through fermion mixing [3841], but also mediated by gauge boson exchange. In our case the leading contribution for the production of a leptophilic vector boson at the LHC is illustrated in figure 1, with the $Z^{\prime}$ emitted from one of the final leptons in Drell-Yan production. The exchanged EW gauge boson can be also a $W$ if $g_{\mathrm{L}}^{\prime}$ is sizable. As a matter of fact, in general the corresponding process with only three charged leptons in the final state plus missing energy provides the most stringent limit on a leptophilic $Z^{\prime}$.

Large collider bounds on the couplings of such a vector boson are in general effectively less restrictive than indirect limits derived from precision experiments and hence, we must review the latter before going on. All processes constraining a leptophilic vector boson involve at least four leptons and two vertices, internal or not. Hence, a small contribution can be the result of a small coupling squared or of one smaller coupling multiplying a larger one. This makes the Large Electron-Positron (LEP) collider constraints very stringent, when they apply. The $Z^{\prime}$ couplings to electrons are mainly constrained by the very precise $e^{+} e^{-} \rightarrow e^{+} e^{-}$LEP data, that very closely follows the SM prediction $[18],{ }^{5}$ leaving no room

the extra U(1) symmetry coupling to muon minus tau lepton number discussed below allows for Yukawa couplings which do preserve the chiral but not the flavor symmetry [35]. But in general, one can build realistic models with extra dimensions or with strong EW symmetry breaking (in which the degree of compositeness of $\mathrm{LH}$ and $\mathrm{RH}$ leptons does not have to be equal) which predict massive vector bosons with flavor and chiral dependent interactions. (See [36, 37] for an example in composite Higgs models in which the RH tau lepton couples differently than the LH one to the extra heavy vector bosons present in the spectrum.) In this case, once they acquire a mass, Yukawa couplings are generated at higher orders in perturbation theory.

${ }^{5}$ The corresponding $95 \%$ Confidence Level (C.L.) bounds are obtained assuming universality but there is no a large flavor dependence [42] and hence, they can be considered a proper estimate of the present upper limits. 
for further improvement at the LHC:

$$
\frac{g_{\mathrm{L}, \mathrm{R}}^{\prime e e}}{M_{Z^{\prime}}}<0.12,0.16 \mathrm{TeV}^{-1}
$$

Similar limits can be derived for $g_{\mathrm{L}, \mathrm{R}}^{\prime e \mu} / M_{Z^{\prime}}$ from $e^{+} e^{-} \rightarrow \mu^{+} \mu^{-}, \tau^{+} \tau^{-}$, respectively. As a matter of fact, the actual limits are slightly more stringent in this case but these bounds are already restrictive enough to make the corresponding vector boson production unobservable at the LHC, as it will be apparent from the analyses of a $Z^{\prime}$ mainly coupling to muons in the next section. On the other hand, bounds on Lepton Flavor Violating (LFV) muon decays like, for instance, $\Gamma(\mu \rightarrow 3 e) / \Gamma(\mu \rightarrow$ all $)<10^{-12}$ at the $90 \%$ C.L. [43] can be fulfilled assuming the diagonal or the off-diagonal coupling to be negligible, $g_{\mathrm{L}, \mathrm{R}}^{\prime e e} / M_{Z^{\prime}}$ or $g_{\mathrm{L}, \mathrm{R}}^{\prime e \mu} / M_{Z^{\prime}}$, in this example. In any case, however, the previous bounds are in practice restrictive enough to neglect them in the study of the LHC and ILC reach for a leptophilic $Z^{\prime}$. Finally, the limit on $\Gamma(\tau \rightarrow 3 \mu) / \Gamma(\tau \rightarrow$ all $)<2.1 \times 10^{-8}$ at the $90 \%$ C.L. [44] implies $g_{\mathrm{L}, \mathrm{R}}^{\prime \mu \mu} g_{\mathrm{L}, \mathrm{R}}^{\prime \mu \tau} / M_{Z^{\prime}}^{2}<10^{-2} \mathrm{TeV}^{-2}$, which can be only satisfied requiring a small enough $g_{\mathrm{L}, \mathrm{R}}^{\prime \mu \tau} / M_{Z^{\prime}}$ because the vector boson must be reconstructed through its decays to muon pairs to be observable at the LHC and then, the diagonal muon couplings can not be too small. Obviously, a proper discussion of all these limits would require to disentangle all possible products of $Z^{\prime}$ couplings contributing to the different processes, also using their angular distributions, but the conclusion would be the same. Hence, only the diagonal $Z^{\prime}$ couplings to muons and taus can be eventually further constrained at the LHC, assuming in either case small enough couplings to electrons in order to satisfy the LEP (and eventually ILC) limits on $e^{+} e^{-} \rightarrow e^{+} e^{-}, \mu^{+} \mu^{-}, \tau^{+} \tau^{-}$. We will denote these four coupling constants with one upper index at most, $g_{\mathrm{L}, \mathrm{R}}^{\prime \mu, \tau}$, from now on.

As already emphasized, in order to observe a leptophilic $Z^{\prime}$ at the LHC one has to sample four-lepton events, $q \bar{q} \rightarrow l \bar{l} l \bar{l}$, as this vector boson must be emitted from a lepton, and decay afterwards into a lepton pair (see figure 1$).{ }^{6}$ Depending on the value of the coupling constants $g_{\mathrm{L}, \mathrm{R}}^{\prime \mu, \tau}, l$ can be a muon or a tau or one of their neutral counterparts. However, only the samples with $4 \mu$ or $3 \mu$ plus missing energy with one $\mu^{+} \mu^{-}$pair reconstructing the $Z^{\prime}$ mass are sensitive to such a vector boson at the LHC, as we shall show. Otherwise, the small branching ratios or the large irreducible backgrounds from gauge boson pair production make the signal unobservable.

We shall discuss in the following how to proceed in order to characterize the new vector boson at the LHC and the ILC. The general case is fixed, as we have stressed by the $Z^{\prime}$ mass, $M_{Z^{\prime}}$, its four couplings to muons and taus, $g_{\mathrm{L}, \mathrm{R}}^{\prime \mu, \tau}$, and its total width, $\Gamma_{Z^{\prime}}$. Obviously, the latter is the sum of its partial decay rates into muons and taus (and their associated neutrinos),

$$
\Gamma_{Z^{\prime}}^{\mu+\tau}=\frac{2 g_{\mathrm{L}}^{\prime \mu 2}+g_{\mathrm{R}}^{\prime \mu 2}+2 g_{\mathrm{L}}^{\prime \tau 2}+g_{\mathrm{R}}^{\prime \tau 2}}{24 \pi} M_{Z^{\prime}}
$$

\footnotetext{
${ }^{6}$ Radiation of EW gauge bosons by one of the final leptons in Drell-Yan production was proposed some time ago to devise new observables which could help to further characterize heavy $Z^{\prime}$ s contributing to this process $[45,46]$ (see also [47]). In our case, however, it is the $Z^{\prime}$ itself which is emitted from the final lepton in Drell-Yan production, whereas the EW gauge bosons are exchanged in the $s$-channel. In both processes the radiated vector boson has the tendency to align along the emitting lepton and hence, opposing to the other one with larger momentum, in general.
} 
and into any other channel which may be open. Thus in general, $\Gamma_{Z^{\prime}} \geq \Gamma_{Z^{\prime}}^{\mu+\tau}$ is an extra free parameter to be determined experimentally, typically adjusting the corresponding Breit-Wigner distribution. As the LHC is only sensitive to the muon signals, as already pointed out and proven in next section, we will only vary $M_{Z^{\prime}}$ and $g_{\mathrm{L}, \mathrm{R}}^{\prime \mu}$ in the corresponding analyses, assuming that $\Gamma_{Z^{\prime}}=\Gamma_{Z^{\prime}}^{\mu}$. In this case the three free parameters can be determined comparing the $4 \mu$ and $3 \mu$ samples. In order to estimate the effect of extra decay channels and hence of a larger $\Gamma_{Z^{\prime}}$, let us discuss, for illustration, the case of the only anomaly-free $Z^{\prime}$ addition in this class of models [35], the combination of muon minus tau LN, $Z_{\mu-\tau}^{\prime}$, with charges $g_{\mathrm{L}, \mathrm{R}}^{\prime \mu, \tau} / g_{\mathrm{L}}^{\prime}$ given by

\begin{tabular}{|l|cccc|}
\hline Multiplet & $L_{\mathrm{L} \mu}=\left(\begin{array}{c}\nu_{\mathrm{L} \mu} \\
\mu_{\mathrm{L}}\end{array}\right)$ & $\mu_{\mathrm{R}}$ & $L_{\mathrm{L} \tau}=\left(\begin{array}{c}\nu_{\mathrm{L} \tau} \\
\tau_{\mathrm{L}}\end{array}\right)$ & $\tau_{\mathrm{R}}$ \\
\hline Charge & 1 & 1 & -1 & -1 \\
\hline
\end{tabular}

In the narrow width approximation (see [48] and references there in), which is a good prescription at the LHC (ILC) up to tens of per cent in the less favorable case of very large vector couplings, this model gives the same predictions for $3 \mu$ plus missing energy and $4 \mu$ production as the model with vanishing couplings to taus but with vector couplings to muons a factor of $\sqrt{2}$ smaller. This reflects the fact that in this approximation the production cross-sections scale as $\Gamma_{Z^{\prime}}^{-1}$ and $\Gamma_{Z^{\prime}}^{\mu+\tau}=2 \Gamma_{Z^{\prime}}^{\mu}$, and this global factor of $1 / 2$ can be absorbed in the redefinition of the strength of the $Z^{\prime}$ emission from one of the final leptons in Drell-Yan production (figure 1). In general, the sign of the charges does not play any role in this case because there is no interference between different final states, and no sign determination is possible either through the production processes studied here. ${ }^{7}$

The couplings for the different cases (set of $Z^{\prime}$ couplings) have been included in a Universal Feynman rules Output (UFO) model [49] by means of FEYNRULES [50, 51]. It can be downloaded from http://cafpe.ugr.es/index.php/pages/other/software. A set of input parameters (Param Cards) for MADGRAPH 5 [52] can be also found there for all $Z^{\prime}$ masses considered in the simulations along the text.

A few comments are in order. The LHC limits for $Z_{\mu-\tau}^{\prime}$ have been recently discussed in the literature $[53,54]$ (see also [55] for the analysis of a related model with leptophilic dark matter) but concentrating on the $4 \mu$ channel, which provides the best bounds only for $g_{\mathrm{R}}^{\prime \mu}$ somewhat larger than $g_{\mathrm{L}}^{\prime \mu}$. Otherwise, the $3 \mu$ plus missing energy channel provides the most stringent limits, as suggested in [56] and we confirm by a detailed analysis in the following. On the other hand, independent bounds from neutrino trident production also impose stringent constraints on $Z_{\mu-\tau}^{\prime}$ and on any other $Z^{\prime}$ model with a non-vanishing coupling $g_{\mathrm{L}}^{\prime \mu}[57,58]$. Nevertheless, they still leave room for further improvement at the LHC and the ILC. In particular, no constraint on $g_{\mathrm{R}}^{\prime \mu}$ is set by $\mu^{+} \mu^{-}$production from the scattering of the muon neutrino off the Coulomb field of a nucleus. The constraints from

\footnotetext{
${ }^{7}$ This is so, however, for the case of a resonant mediator of the leptophilic interaction and when the SM background is relatively small but not, for example, for SM extensions involving four-lepton operators, as we shall discuss in the following. We will find, for instance, that their interference with the SM background makes the sign of their coefficients physically meaningful.
} 
$g-2$ are weaker but also apply to the RH muon coupling $[54,57,58]$. Finally, we focus our attention on $Z^{\prime}$ masses above the $Z$ mass, as we want to investigate the ultimate reach at large colliders. Masses below $M_{Z}$ have been considered in $[54,55,57,58]$.

In next section we study the LHC reach for a leptophilic $Z^{\prime}$. We derive discovery and exclusion limits as a function of its mass, $M_{Z^{\prime}}$, and its LH coupling to muons, $g_{\mathrm{L}}^{\prime} \equiv g_{\mathrm{L}}^{\prime \mu}$, for different values of the corresponding $\mathrm{RH}$ coupling, $\xi \equiv g_{\mathrm{R}}^{\prime \mu} / g_{\mathrm{L}}^{\prime \mu}$. Such a vector boson could be eventually excluded at the $\mathrm{LHC}^{8}$ for $Z^{\prime}$ masses up to $\sim 1 \mathrm{TeV}$ and $g_{\mathrm{L}}^{\prime}$ and $\xi$ of order one in the high-luminosity phase with an integrated luminosity $\mathcal{L}_{\text {int }}=3 \mathrm{ab}^{-1}$ and a CME $\sqrt{s}=14 \mathrm{TeV}$. If a leptophilic $Z^{\prime}$ below this mass is discovered, its couplings can be fully determined up to a global normalization proportional to the inverse of the square root of the total $Z^{\prime}$ width, $\Gamma_{Z^{\prime}}^{-1 / 2}$, which has to be measured independently from the invariant $\mu^{+} \mu^{-}$mass distribution reconstructing the $Z^{\prime}$ mass. The codes used and the cuts applied are described in the corresponding section. The analyses to study the corresponding ILC reach are described in section 3. In this case there is no $W$ exchange contribution but the $3 \mu$ plus missing energy sample is traded by the $2 \mu$ plus missing energy one, obtaining at the end bounds comparable to those derived at the LHC but only for low enough $Z^{\prime}$ masses (smaller than the ILC CME $\sqrt{s}=500 \mathrm{GeV}$ ) and for an integrated luminosity $\mathcal{L}_{\text {int }}=500 \mathrm{fb}^{-1}$. However, at the ILC not only the $Z^{\prime}$ total width is expected to be measurable with a better precision but in contrast with the LHC, the $Z^{\prime}$ couplings to taus can be constrained analyzing the $2 \mu$ plus missing energy and $2 \mu 2 \tau$ final states, too. Finally, in section 4 we comment on the limit of very large $M_{Z^{\prime}}$ and arbitrary effective four-lepton interactions. Although bounds on their coefficients can be also derived from the corresponding production cross-sections, they are too weak to allow for a resonance interpretation. Section 5 is devoted to conclusions and final remarks.

\section{$2 \quad$ LHC reach for a leptophilic $Z^{\prime}$}

As argued in the Introduction, current experimental constraints set stringent limits on the coupling of a leptophilic $Z^{\prime}$ to electrons or to a pair of leptons of different flavor. Thus the LHC can be only sensitive, a priori, to its diagonal couplings to muons and taus. As a matter of fact, only the couplings to muons can be determined at the LHC because the SM backgrounds are too large for taus to make any conclusive claim. We present in this section a detailed simulation analysis to estimate the LHC potential for discovering or excluding a new heavy neutral vector boson of mass $M_{Z^{\prime}}$ coupling to the LH muon doublet with strength $g_{\mathrm{L}}^{\prime}$ and to the $\mathrm{RH}$ muon singlet with strength $\xi g_{\mathrm{L}}^{\prime}$. Its total width reads

$$
\Gamma_{Z^{\prime}}=\frac{g_{\mathrm{L}}^{\prime 2}\left(2+\xi^{2}\right)+W^{i \neq \mu}}{24 \pi} M_{Z^{\prime}}
$$

where $W^{i \neq \mu}$ takes into account all decay channels different than $\mu^{+} \mu^{-}$and $\bar{\nu}_{\mu} \nu_{\mu}$.

The main production mechanism is the one depicted in figure 1 with the $Z^{\prime}$ radiated by one of the final muons (neutrinos) in Drell-Yan production. The production cross-section

\footnotetext{
${ }^{8}$ The limits derived using Run I data are much weaker than the indirect bounds from neutrino trident production, $\nu N \rightarrow \nu \mu^{+} \mu^{-} N$.
} 
for such a (leptophilic) $Z^{\prime}$ is relatively small and suppressed for heavy vector boson masses $M_{Z^{\prime}}$. Depending on the initial parton state the exchanged EW gauge boson can be neutral $(\gamma, Z)$ and the final mode $4 \mu$ or $2 \mu 2 \nu_{\mu}$, or charged $(W)$ and the final mode $3 \mu 1 \nu_{\mu}$. Only events with at least two muons are considered because we assume that $Z^{\prime} \rightarrow \mu^{+} \mu^{-}$in order to allow for the vector boson reconstruction, which is compulsory to enhance the signal to background ratio to an observable level. In practice, we only consider the two most promising channels: $3 \mu$ plus missing energy and $4 \mu$.

We have implemented the model in a UFO format [49] using FEYNRULES [50, 51]. Partonlevel events have been generated with MADGRAPH 5 [52] and showered/hadronized with PYTHIA 6 [59]. Detector effects have been simulated with DELPHES 3 [60] and the experimental analyses performed by means of MADANALYSIS $5[61,62]$. Jets are reconstructed with the anti- $k_{t}$ algorithm with $R=0.5$, as implemented in FASTJET [63].

The applied cuts are collected in tables 1 and 2 for the $3 \mu$ plus missing energy and $4 \mu$ final states, respectively. The $3 \mu$ plus missing energy analysis closely follows the one in [64], which we found to be the most constraining among the current experimental searches. Still, current constraints are much weaker than the indirect ones from the neutrino trident process $[57,58]$ and hence, we shall not report detailed results at $\sqrt{s}=8 \mathrm{TeV}$. As an example we have run the analysis in [64] for a $Z_{\mu-\tau}^{\prime}$ with mass of $140 \mathrm{GeV}$ and coupling $g_{\mathrm{L}}^{\prime}=0.3$ (at the verge of the trident exclusion bound). This analysis is similar to the one presented in table 1 except that it does not include the last (Mass window) cut and the numerical values of the constraints are somewhat less stringent. The current bound on the observed number of events reported in the region named "SRnoZc" in [64] (the most sensitive to our signal) is 6.8 whereas we obtain that only 1.5 of our signal events pass those cuts. A simple modification of the experimental analysis requiring only muons in the final state (as opposed to muons and electrons) and including the last (Mass window) cut would reduce the background to an almost negligible level but also our signal which would be too small to provide a significant limit. The situation is even worse in the $4 \mu$ channel in which the cross sections are even smaller. Nevertheless, we have used the $3 \mu$ plus missing energy analysis to validate our generator implementation, finding good agreement. In all simulations we have only considered the irreducible backgrounds, which are by far the most relevant ones, unless otherwise stated, renormalizing our results with a global factor to account for non-irreducible ones as well as for higher-order effects. In order to be conservative, this factor is applied to the estimation of the background but not to the signal. Finally, an important observation is that it would be extremely useful that the experimental collaborations present their results separately for electrons and muons as only the latter contribute to our signal.

We have generated and analyzed signal events for different masses and couplings, as well as irreducible SM backgrounds, always aiming at generating samples with an integrated luminosity about five times larger than the target one of $3 \mathrm{ab}^{-1}$ in order to reduce the Monte Carlo uncertainties. Once the events have been analyzed, we use the $C L_{s}$ [65] method to obtain $95 \%$ C.L. bounds on the corresponding $Z^{\prime}$ signal. To assess the discovery 


\begin{tabular}{|ll|}
\hline Basic cuts & $p_{\mathrm{T}}^{\ell}>50 \mathrm{GeV},\left|\eta_{\ell}\right|<2.4, \Delta R(j \ell)>0.4, p_{\mathrm{T}}^{j}>20 \mathrm{GeV},\left|\eta_{j}\right|<2.5$ \\
\hline b veto & No b jets \\
Number of muons & $N_{\mu}=3$ (net charge $= \pm 1$ ) \\
Low mass resonance veto & $m_{\mu^{+} \mu^{-}}>12 \mathrm{GeV}$ \\
$Z$ veto & $\left|m_{\mu^{+} \mu^{-}}-M_{Z}\right|>10 \mathrm{GeV}$ \\
Missing $E_{\mathrm{T}}$ & $E_{\mathrm{T}}>100 \mathrm{GeV}$ \\
Transverse mass & $m_{\mathrm{T}}>110 \mathrm{GeV}$ \\
Mass window & $\left|m_{\mu^{+} \mu^{-}}-M_{Z^{\prime}}\right|<0.1 M_{Z^{\prime}}$ \\
\hline
\end{tabular}

Table 1. Cuts for $3 \mu$ plus missing energy events. The transverse mass is computed with the transverse missing energy and the lepton not belonging to the pair which better reconstructs the $Z^{\prime}$ boson mass. Only isolated muons are considered.

\begin{tabular}{|ll|}
\hline Basic cuts & $p_{\mathrm{T}}^{\ell}>30 \mathrm{GeV}, \eta_{\ell}<2.4, \Delta R(j \ell)>0.4$ \\
\hline Number of muons & $N_{\mu}=4$ (zero net charge) \\
muon spectrum & $p_{\mathrm{T}}^{\mu_{1,2,3}}>100,80,60 \mathrm{GeV}$ \\
$Z$ veto & $\left|m_{\mu^{+} \mu^{-}}-M_{Z}\right|>10 \mathrm{GeV}$ \\
Mass window & $\left|m_{\mu^{+} \mu^{-}}-M_{Z^{\prime}}\right|<0.1 M_{Z^{\prime}}$ \\
\hline
\end{tabular}

Table 2. Cuts for $4 \mu$ events. Only isolated muons are considered.

potential we use $\mathcal{S}=5$ with

$$
\mathcal{S}(s, b)=\sqrt{2\left((s+b) \log \left(1+\frac{s}{b}\right)-s\right)},
$$

which gives accurate results for the Monte Carlo statistics used [66]. In the above equation $s$ and $b$ stand for the number of signal and background events, respectively.

In figure 2 we draw the LHC $5 \sigma$ discovery (dashed curve) and the $95 \%$ C.L. exclusion (solid curve) limits for a $Z^{\prime}$ only coupling to LH muons as a function of its mass for $3 \mu$ plus missing energy (left) and $4 \mu$ (right) events. Thick (thin) curves represent the results for an integrated luminosity of $3 \mathrm{ab}^{-1}\left(300 \mathrm{fb}^{-1}\right)$. We also show the bound from the neutrino trident production (dotted line) $[57,58]$ taking into account CHARM-II and CCFR data, for comparison. This bound compares with arbitrary $\xi$ curves because it does not depend on this parameter. Other bounds, like the one from $g-2$ are much weaker, although they involve both couplings and become more stringent for larger $\xi$. As we can see in the figures, current constraints leave some room (mainly in the $3 \mu$ plus missing energy channel) for discovery even with the limited integrated luminosity of $300 \mathrm{fb}^{-1}$. Masses up to $\sim 1 \mathrm{TeV}$ can be probed for $g_{\mathrm{L}}^{\prime}$ of order one in both channels at the high-luminosity phase of the LHC.

Obviously, the LHC limits also depend on the RH coupling to muons. In figure 3 we show the corresponding exclusion limits implied by the non-observation of a departure from the SM prediction in the $3 \mu$ plus missing energy (left panel) and $4 \mu$ (right panel) channels, as a function of the vector boson mass $M_{Z^{\prime}}$ and the $Z^{\prime}$ coupling to LH muons 

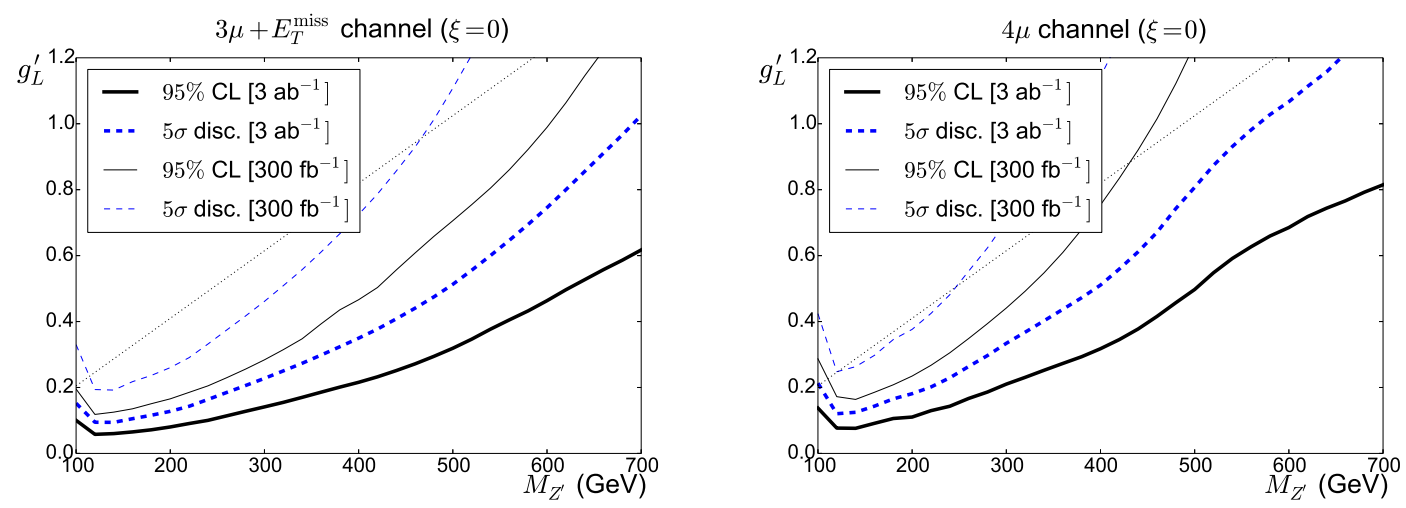

Figure 2. Discovery and exclusion limits for a leptophilic $Z^{\prime}$ only coupling to LH muons as a function of its mass at the LHC. We also draw the bounds from neutrino trident production (straight line), for comparison (see the text for details).
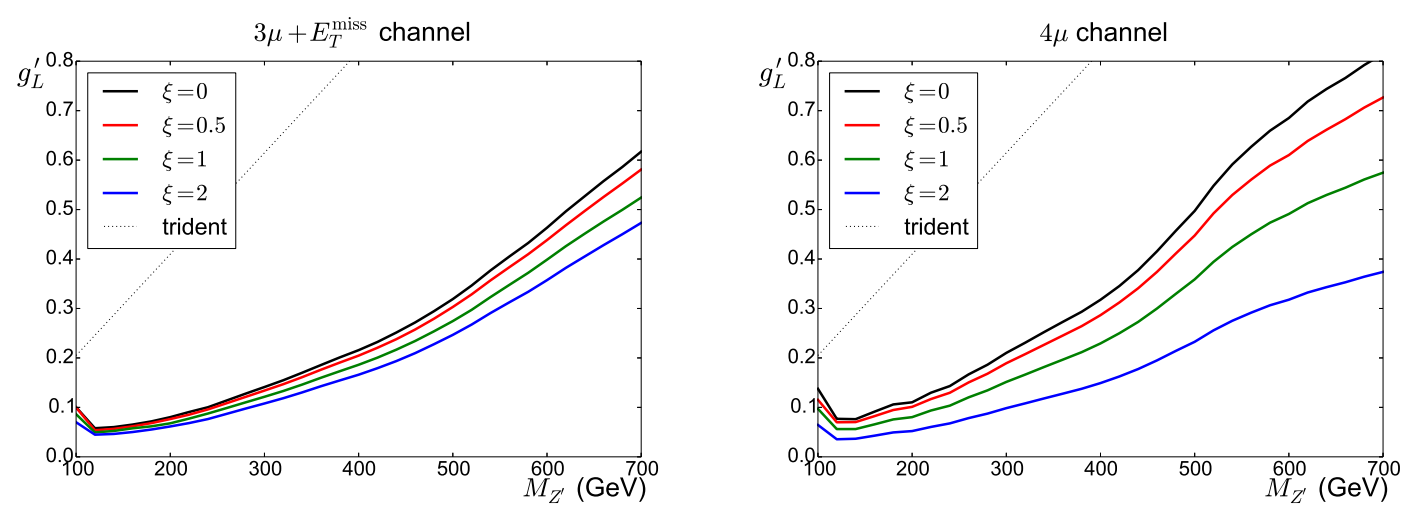

Figure 3. $95 \%$ C.L. exclusion limits for a leptophilic $Z^{\prime}$ as a function of its mass and its coupling to LH muons for different values of its coupling to their RH counterparts at the LHC in the $3 \mu$ plus missing energy (left) and $4 \mu$ (right) channels. The different lines correspond to $\xi=0,0.5,1$ and 2 , from top to bottom, respectively. The bound from neutrino trident production (dotted line) is also shown for comparison.

$g_{\mathrm{L}}^{\prime}$ for different values of the $\mathrm{RH}$ coupling $\xi g_{\mathrm{L}}^{\prime}$. These limits improve with the value of the coupling to $\mathrm{RH}$ muons $\xi g_{\mathrm{L}}^{\prime}$ in both cases; but much faster for the neutral final state because the two exchanged EW gauge bosons, $\gamma$ and $Z$, also couple to $\mathrm{RH}$ muons, which is not the case for the charged final state which requires the exchange of a $W$ gauge boson which only couples to LH fermions. (In this case the contribution proportional to $g_{\mathrm{R}}^{\prime}$ is suppressed by the ratio of the muon over the $Z^{\prime}$ mass, being therefore negligible.) We assume in all cases that there are no other $Z^{\prime}$ decay channels open. The $2 \mu 2 \nu_{\mu}$ sample is dominated by the irreducible background.

As it is apparent from figures 2 and 3 , the limits for the charged final state are more stringent than for the neutral one, except for somewhat larger $Z^{\prime}$ coupling to $\mathrm{RH}$ than to $\mathrm{LH}$ muons and hence, for relatively larger $4 \mu$ cross-sections. It is also evident that the dependence on $\xi$ is larger in the $4 \mu$ case as indicated by the bound variation. This, in particular, 
means that this coupling ratio can be measured comparing the number of events in both channels. Obviously, the observed global strength of the $Z^{\prime}$ couplings, $g_{\mathrm{L}}^{\prime}$, depends on the total $Z^{\prime}$ width, and the latter must be measured to determine the former. All these comments can be made quantitative running the corresponding Monte Carlo simulation but in this case it easy to prove these results analytically, too. Indeed, different final states do not interfere and in practice neither the two diagrams contributing to each process, because we require the reconstruction of the new gauge boson which is in general rather narrow. Hence, we can approximate the corresponding cross-sections assuming the narrow width approximation (which is good up to at most $20 \%$ when we compare to the numerical simulation):

$$
\sigma_{Z^{\prime}}\left(p p \rightarrow X \mu^{+} \mu^{-}\right) \approx \sigma_{X Z^{\prime}}\left(M_{Z^{\prime}}\right) \operatorname{Br}\left(Z^{\prime} \rightarrow \mu^{+} \mu^{-}\right),
$$

and extrapolating the behavior of the diagram in figure 1 we find:

$$
\begin{aligned}
\sigma_{Z^{\prime}}\left(p p \rightarrow 3 \mu+E_{\mathrm{T}}\right) & \approx f_{3 \mu+E_{\mathrm{T}}}^{W}\left(M_{Z^{\prime}}\right) g_{\mathrm{L}}^{\prime 2} \frac{g_{\mathrm{L}}^{\prime 2}\left(1+\xi^{2}\right)}{g_{\mathrm{L}}^{\prime 2}\left(2+\xi^{2}\right)+W^{i \neq \mu}} \\
\sigma_{Z^{\prime}}(p p \rightarrow 4 \mu) & \approx f_{4 \mu}^{\gamma+Z}\left(M_{Z^{\prime}}\right) g_{\mathrm{L}}^{\prime 2}\left(2.5+\xi^{2}\right) \frac{g_{\mathrm{L}}^{\prime 2}\left(1+\xi^{2}\right)}{g_{\mathrm{L}}^{\prime 2}\left(2+\xi^{2}\right)+W^{i \neq \mu}}
\end{aligned}
$$

The fraction stands for the $Z^{\prime}$ branching ratio (Br) into muons (see eqs. (1.3) and (2.1)), and the $Z^{\prime}$ coupling dependence is derived neglecting the EW gauge boson masses and assuming that the cross-section is dominated by the $\bar{u} u$ partonic contribution, as suggested by the proton content and the EW couplings. We have numerically computed the exact factor without any of these approximations and found an excellent agreement, up to a negligible dependence on $m_{\mu^{+} \mu^{-}}$. We have also made an extra non-trivial check of the validity of eqs. (2.4) by plotting the exclusion limits reported in figure 3 scaled by the $\xi$ dependence in eqs. (2.4). The almost perfect match is shown in figure 4 . The near equality of $f_{3 \mu+E_{\mathrm{T}}}^{W}\left(M_{Z^{\prime}}\right)$ and $f_{4 \mu}^{\gamma+Z}\left(M_{Z^{\prime}}\right)$ after selection cuts is, however, accidental.

Once we are convinced of the goodness of the quantitative analytic results, we can attempt to determine $\xi$ from the number of events with $3 \mu$ plus missing energy and $4 \mu$ reconstructing a $Z^{\prime} \rightarrow \mu^{+} \mu^{-}$. This is done in figure 5 , where we show the corresponding cross-section ratio:

$$
\left(2.5+\xi^{2}\right) \frac{\sigma_{Z^{\prime}}\left(p p \rightarrow 3 \mu+E_{\mathrm{T}}^{\mathrm{miss}}\right)}{\sigma_{Z^{\prime}}(p p \rightarrow 4 \mu)} \approx 9.4
$$

before and after cuts, as a function of the $Z^{\prime}$ mass. Obviously, the approximation improves with the new vector boson mass and depends on the cuts, but it is clear that the ratio of the number of charge and neutral events is a sensitive probe of the value of $\xi$. The determination of the $Z^{\prime}$ width will also allow for the measurement of the global strength of the $Z^{\prime}$ couplings, $g_{\mathrm{L}}^{\prime}$. This, however, does not seem to be easy at the LHC for the muon momentum measurement degrades for large values. In figure 6 we plot the observed vector boson width ${ }^{9} \sim 60 \mathrm{GeV}$ for a $Z^{\prime}$ with a mass of $500 \mathrm{GeV}$ and a total width of $10 \mathrm{GeV}$. Short

\footnotetext{
${ }^{9}$ Experimentalists will certainly do better but this measurement is difficult due to the larger uncertainty associated to the determination of large muon momenta, as well as to the tendency of the $Z^{\prime}$ to align with the emitting lepton and the relatively small number of signal events.
} 


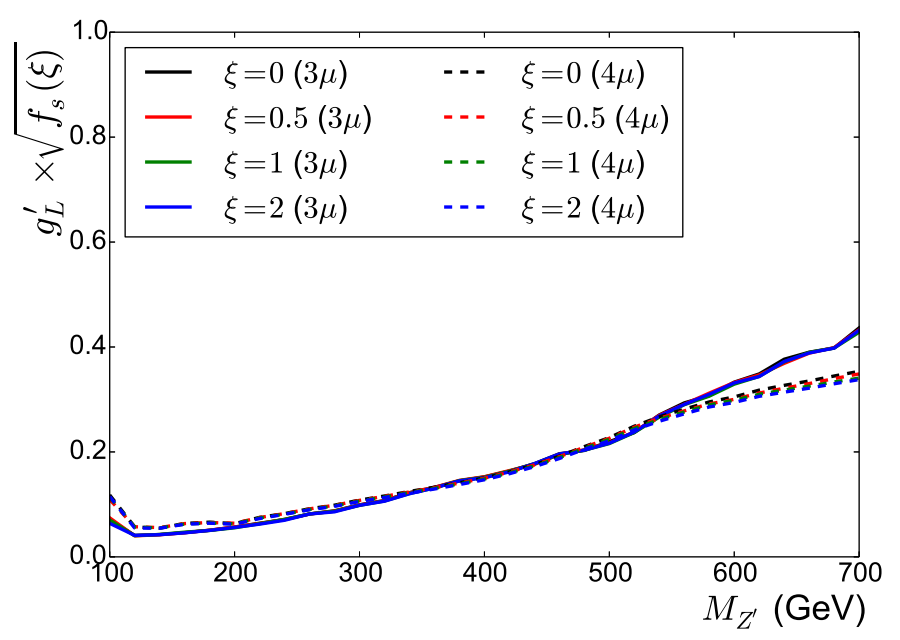

Figure 4. $95 \%$ C.L. exclusion limits for a leptophilic $Z^{\prime}$ as a function of its mass and its coupling to LH muons for different values of its coupling to their RH counterparts at the LHC, corrected for the new vector boson coupling dependence in eqs. (2.4): $f_{3 \mu}(\xi)=\frac{1+\xi^{2}}{2+\xi^{2}}$ and $f_{4 \mu}(\xi)=\left(2.5+\xi^{2}\right) \frac{1+\xi^{2}}{2+\xi^{2}}$.

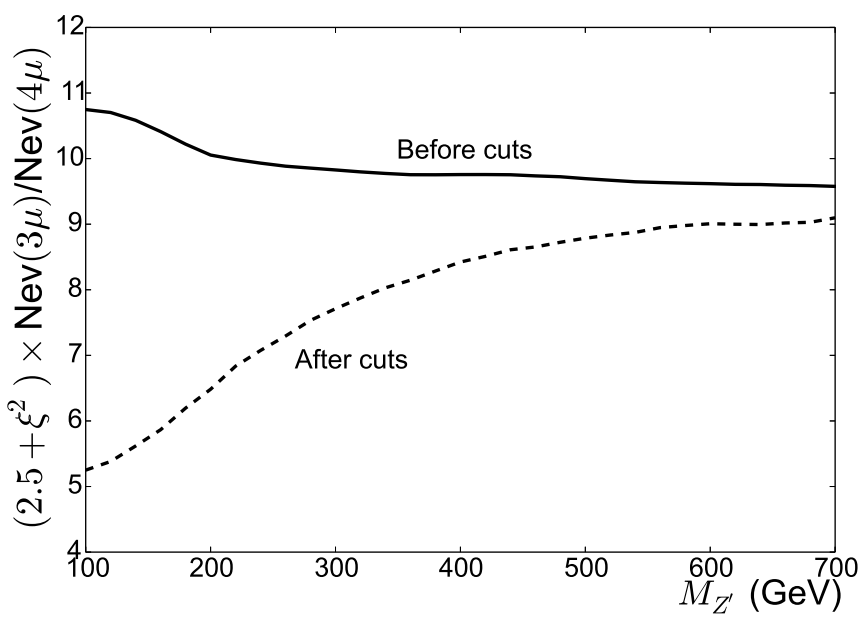

Figure 5. Ratio of $3 \mu$ plus missing energy to $4 \mu$ events as a function of the $Z^{\prime}$ mass at the LHC. The quite different values before and after selection cuts are mainly due to the sensitivity to the cut on the invariant mass of the muon pair reconstructing the vector boson. 


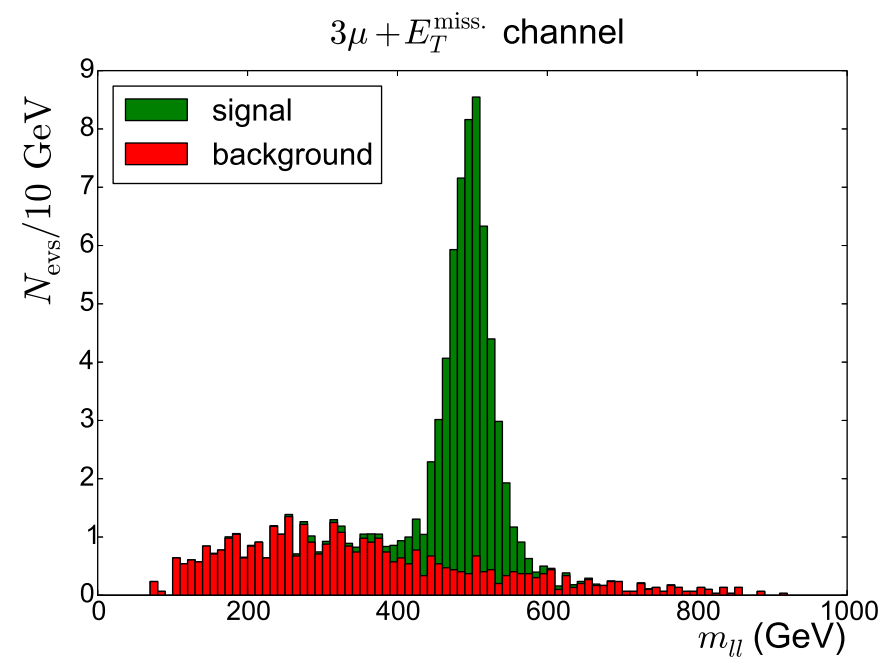

Figure 6. $Z^{\prime}$ mass reconstruction in the $3 \mu$ plus missing energy channel for $M_{Z^{\prime}}=500 \mathrm{GeV}$, $g_{\mathrm{L}}^{\prime}=0.7$ and $\xi=1$, with $W^{i \neq \mu}=0$.

of a precise measurement of the $Z^{\prime}$ width we can still use the fact that $W^{i \neq \mu} \geq 0$ to obtain a lower bound on $g_{\mathrm{L}}^{\prime}$ from eqs. (2.4). Other observables like, for instance, charged asymmetries can be also measured, but all the model dependence stays described by eqs. (2.4).

Given the relatively small cross-sections, the additional branching ratios and the missing energy accompanying any tau decay, it will be difficult to conclude anything using leptophilic final states involving tau leptons. Let us illustrate this in the anomaly-free muon minus tau LN case, $\mathrm{L}_{\mu}-\mathrm{L}_{\tau},{ }^{10}$ for $2 \mu \tau_{\mathrm{h}} \nu_{\tau}$ events, where $\tau_{\mathrm{h}}$ stands for a tau lepton decaying hadronically. Even in this mode with the handle of an opposite-sign muon pair reconstructing the $Z_{\mu-\tau}^{\prime}$ mass, the SM backgrounds remain problematic. For example, in this channel the number of signal events is $\sim 51 \times g_{\mathrm{L}}^{\prime 2}$ for $M_{Z_{\mu-\tau}^{\prime}}=300 \mathrm{GeV}$ and an integrated luminosity of $3 \mathrm{ab}^{-1}$. The number of $\bar{t} t$ events which passes our selection cuts is $\sim 228$, where we have assumed that the probability for a jet to be misidentified as a tau lepton decaying hadronically is $10^{-3}$, which is rather conservative, and a $k$-factor equal to 1.9 for $\bar{t} t$ production. (Irreducible SM backgrounds with several EW gauge bosons are negligible.) The applied cuts are the same as in table 1 , but with $p_{\mathrm{T}}^{\tau_{\mathrm{h}}}(>20 \mathrm{GeV})$ the lepton momentum used to compute the transverse mass and $\left|\eta_{\tau_{\mathrm{h}}}\right|<2.4$. Just considering the $\bar{t} t$ background we need 31 (80) signal events to set the $95 \%(5 \sigma)$ exclusion (discovery) limit. This translates into $g_{\mathrm{L}}^{\prime}<0.78(1.25)$ for $M_{Z_{\mu-\tau}^{\prime}}=300 \mathrm{GeV}$, above the trident bound in both cases (see figure 3). Thus, we must rely on an experimental performance (analysis) much better that this estimate to extract any information on leptophilic final states with tau leptons.

\footnotetext{
${ }^{10}$ The limits in figure 3 for $\xi=1$ also apply to this case but scaling the corresponding curve up by a factor $\sim \sqrt{2}$, to take into account for the $Z_{\mu-\tau}^{\prime}$ total width $\left(W^{i \neq \mu} \equiv W^{\tau}=W^{\mu}=g_{\mathrm{L}}^{\prime 2}\left(2+\xi^{2}\right)\right.$ in eqs. (2.1) and (2.4)).
} 


\section{$3 \quad$ ILC reach for a leptophilic $Z^{\prime}$}

As we will argue in the following, it will be eventually possible to measure the tau couplings of a leptophilic $Z^{\prime}$ at the ILC, but not at the LHC as just illustrated in the previous section. In this sense, both machines are complementary, although no measurement of a leptophilic vector boson can be done at the ILC if such a $Z^{\prime}$ is not previously observed at the LHC.

The ILC production mechanism for a leptophilic vector boson with vanishing coupling to electrons is the same as at the LHC in figure 1 but with the initial strong interacting partons replaced by an $e^{+} e^{-}$pair. The size of the cross-section is of the same order in both machines and the phenomenological search similar, too. However, at the ILC the initial state is neutral and only the neutral EW gauge bosons, $\gamma$ and $Z$, can be exchanged in the $s$-channel, although the three possible final states, $4 \mu, 2 \mu 2 \nu_{\mu, \tau}$ and $2 \mu 2 \tau$, with a muon pair reconstructing the leptophilic vector boson, $Z^{\prime} \rightarrow \mu^{+} \mu^{-}$, emerge over the background in this case. Moreover, using in the last two channels, $2 \mu 2 \nu_{\mu, \tau}$ and $2 \mu 2 \tau$, the two muon momenta and the total momentum, $P$, to reconstruct the new vector boson invariant mass, $M_{Z^{\prime}}^{2}=\left(P-p_{\mu^{+}}-p_{\mu^{-}}\right)^{2}$, we can sample the four-lepton events with invisible $Z^{\prime}$ decays, $Z^{\prime} \rightarrow \bar{\nu} \nu$, in the first case and the four-lepton events with the new vector boson decaying into taus, $Z^{\prime} \rightarrow \tau^{+} \tau^{-}$, in the second one and hence, define two other differentiated sets of events (processes). Thus, we will deal with five different samples at the ILC, with the tau leptons identified by their hadronic decays, $\tau_{\mathrm{h}}$. The measurement of the corresponding cross-sections could, a priori, overdetermine the $Z^{\prime}$ couplings to $\mathrm{LH}$ and $\mathrm{RH}$ muons, $g_{\mathrm{L}}^{\prime}$ and $\xi g_{\mathrm{L}}^{\prime}$, as well as to $\mathrm{LH}$ and $\mathrm{RH}$ taus, $g_{\mathrm{L}}^{\prime \tau}$ and $\xi_{\tau} g_{\mathrm{L}}^{\prime \tau}$, once the total $Z^{\prime}$ width (eqs. (1.3) and (2.1)) is determined fitting the corresponding Breit-Wigner distribution to the lepton pairs reconstructing the $Z^{\prime}$ mass. However, in practice, as we shall make explicit below, the similar coupling dependence of the two $2 \mu 2 \nu_{\mu, \tau}$ subsamples on one hand and of the two $2 \mu 2 \tau$ ones on the other, which is accidental and a consequence of the particular value of the SM mixing angle, only allows for the determination of three coupling constant combinations. But the two tau couplings enter in two of them and with different dependence and hence, even with large errors due to the small cross-sections involved, both can be determined at the ILC. In any case, the muon couplings can be more precisely measured at the LHC, but not the $Z^{\prime}$ total width for low vector boson masses, which are the only accessible at the ILC.

Applying the same approximations as for the LHC in the former section, we can also extrapolate the corresponding cross-sections at the ILC, obtaining:

$$
\begin{aligned}
\sigma_{Z^{\prime} \rightarrow \mu^{+} \mu^{-}}\left(e^{+} e^{-} \rightarrow 4 \mu\right) & \approx f_{4 \mu}^{\prime \gamma+Z}\left(M_{Z^{\prime}}\right) g_{\mathrm{L}}^{\prime 2}\left(1.15+\xi^{2}\right) \frac{g_{\mathrm{L}}^{\prime 2}\left(1+\xi^{2}\right)}{g_{\mathrm{L}}^{\prime 2}\left(2+\xi^{2}\right)+W^{i \neq \mu}}, \\
\sigma_{Z^{\prime} \rightarrow \mu^{+} \mu^{-}}\left(e^{+} e^{-} \rightarrow 2 \mu+E_{\mathrm{T}}\right) & \approx f_{2 \mu+E_{\mathrm{T}}^{\prime Z}}^{\prime Z}\left(M_{Z^{\prime}}\right)\left(g_{\mathrm{L}}^{\prime 2}+g_{\mathrm{L}}^{\prime \tau 2}\right) \frac{g_{\mathrm{L}}^{\prime 2}\left(1+\xi^{2}\right)}{g_{\mathrm{L}}^{\prime 2}\left(2+\xi^{2}\right)+W^{i \neq \mu}}, \\
\sigma_{Z^{\prime} \rightarrow \bar{\nu} \nu}\left(e^{+} e^{-} \rightarrow 2 \mu+E_{\mathrm{T}}\right) & \approx f_{2 \mu+E_{\mathrm{T}}}^{\prime \gamma+Z}\left(M_{Z^{\prime}}\right) g_{\mathrm{L}}^{\prime 2}\left(1.15+\xi^{2}\right) \frac{g_{\mathrm{L}}^{\prime 2}+g_{\mathrm{L}}^{\prime \tau 2}}{g_{\mathrm{L}}^{\prime 2}\left(2+\xi^{2}\right)+W^{i \neq \mu}}, \\
\sigma_{Z^{\prime} \rightarrow \mu^{+} \mu^{-}}\left(e^{+} e^{-} \rightarrow 2 \mu+2 \tau\right) & \approx f_{2 \mu+2 \tau}^{\prime \gamma+Z}\left(M_{Z^{\prime}}\right) g_{\mathrm{L}}^{\prime \tau 2}\left(1.15+\xi_{\tau}^{2}\right) \frac{g_{\mathrm{L}}^{\prime 2}\left(1+\xi^{2}\right)}{g_{\mathrm{L}}^{\prime 2}\left(2+\xi^{2}\right)+W^{i \neq \mu}},
\end{aligned}
$$




$$
\sigma_{Z^{\prime} \rightarrow \tau^{+} \tau^{-}}\left(e^{+} e^{-} \rightarrow 2 \mu+2 \tau\right) \approx f_{2 \mu+2 \tau}^{\prime \gamma+Z}\left(M_{Z^{\prime}}\right) g_{\mathrm{L}}^{\prime 2}\left(1.15+\xi^{2}\right) \frac{g_{\mathrm{L}}^{\prime 2}\left(1+\xi_{\tau}^{2}\right)}{g_{\mathrm{L}}^{\prime 2}\left(2+\xi^{2}\right)+W^{i \neq \mu}}
$$

where if the tau couplings are non-vanishing, $W^{i \neq \mu}$ includes at least the tau contribution $g_{\mathrm{L}}^{\prime \tau 2}\left(2+\xi_{\tau}^{2}\right)$. As pointed out, the second and third equations have a very similar coupling constant dependence due to the particular value of the Weinberg angle, which results in the term 1.15 in the third cross-section to be compared with the unit term in the branching ratio in the second one; and analogously for the fourth and fifth equations. Although the muon couplings can be measured at the LHC, both tau couplings can be only determined at the ILC, providing a new example of the complementarity of both colliders. After cuts all (sub)samples will differ, except for the last two which we will analyze (add) together. Before selection cuts, however,

$$
f_{4 \mu}^{\prime \gamma+Z}\left(M_{Z^{\prime}}\right) \approx 2 f_{2 \mu+E_{\mathrm{T}}}^{\prime Z}\left(M_{Z^{\prime}}\right) \approx f_{2 \mu+E_{\mathrm{T}}^{\prime}}^{\prime \gamma+Z}\left(M_{Z^{\prime}}\right) \approx f_{2 \mu+2 \tau}^{\prime \gamma+Z}\left(M_{Z^{\prime}}\right)
$$

In order to estimate the ILC reach for different samples we have followed the same generation procedure as for the LHC. Hadronic taus, $\tau_{\mathrm{h}}$, are tagged by a pure geometrical method, becoming a jet a potential hadronic tau if a generated tau is found within a fixed distance $\Delta R=0.5$ of the jet axis, with an efficiency of 0.5 . For each particular sample, we impose a different set of cuts in order to isolate the signal from the background. They also allow for discriminating between the different signal samples. The cuts are shown in table 3 , and have been implemented using MADANALYSIS 5 [61,62]. From top to bottom, they refer to the samples $4 \mu, 2 \mu 2 \nu_{\mu, \tau}$ with $Z^{\prime}$ decaying into muons, $2 \mu 2 \nu_{\mu, \tau}$ with $Z^{\prime}$ decaying into neutrinos and $2 \mu 2 \tau$ with $Z^{\prime}$ decaying into both muons and taus, with at least one tau lepton decaying hadronically. In the last case we sum both subsamples to improve the statistics because their model dependence and the efficiency in the sampling are very similar. $m_{\bar{\nu} \nu}$ and $m_{\tau^{+} \tau^{-}}$stand for the invariant mass reconstructed from the two observed muons and the initial momentum, $\sqrt{\left(P-p_{\mu^{+}}-p_{\mu^{-}}\right)^{2}}$.

Only irreducible backgrounds are considered for each case. We have checked that other backgrounds are negligible after applying the cuts in table 3 . In particular, in the $2 \mu 2 \tau$ case $Z+$ jets is subleading given the small fake-rate for tau tagging (of around $10^{-3}$ ).

Analogously as for the LHC, in figure 7 we plot the ILC discovery $(5 \sigma)$ and exclusion (95\% C.L.) limits for a leptophilic $Z^{\prime}$ as a function of its mass and coupling to LH muons using only $4 \mu$ events. ( $Z^{\prime}$ couplings to taus are neglected if not stated otherwise.) The bounds from neutrino trident production are also shown, for comparison (see the former section for details). ${ }^{11}$ Obviously, although the ILC limits are similar to the LHC ones for low $Z^{\prime}$ masses, the bounds rapidly deteriorate for $Z^{\prime}$ masses near the ILC CME (compare figures 2 and 3 with 7 ).

Also similarly as for the LHC, we want to test how good the approximations in eqs. (3.1) are, and how well the $Z^{\prime}$ properties can be determined. Thus, in figure 8 we draw, for instance, the exclusion limits for each four-lepton subsample scaled by the coupling dependence in eqs. (3.1), ${ }^{12}$ obtaining again an almost perfect matching. To measure the tau

\footnotetext{
${ }^{11}$ The limits from $g-2$ are less stringent when comparable (see refs. [54-58]).

${ }^{12}$ As the $Z^{\prime}$ is assumed not to couple to taus in this case, only the first three processes and equations are relevant.
} 


\begin{tabular}{|ll|}
\hline Basic cuts & $p_{T}^{\ell}>10 \mathrm{GeV},\left|\eta_{\ell}\right|<2.47, \Delta R(j \ell)>0.4, p_{T}^{j}>20 \mathrm{GeV},\left|\eta_{j}\right|<2.5$ \\
\hline Number of muons & $N_{\mu}=4$ (zero net charge) \\
$Z$ veto & $\left|m_{\mu^{+} \mu^{-}}-M_{Z}\right|>10 \mathrm{GeV}$ \\
Mass window & $\left|m_{\mu^{+} \mu^{-}}-M_{Z^{\prime}}\right|<10 \mathrm{GeV}$ \\
\hline Number of muons & $N_{\mu}=2$ (zero net charge) \\
Number of taus & $N_{\tau_{\mathrm{h}}}=0$ \\
$Z$ veto & $\left|m_{\mu^{+} \mu^{-}}-M_{Z}\right|$ and $\left|m_{\bar{\nu} \nu}-M_{Z}\right|>10 \mathrm{GeV}$ \\
Mass window & $\left|m_{\mu^{+} \mu^{-}}-M_{Z^{\prime}}\right|<10 \mathrm{GeV}$ \\
\hline Number of muons & $N_{\mu}=2($ zero net charge $)$ \\
Number of taus & $N_{\tau_{\mathrm{h}}}=0$ \\
$Z$ veto & $\left|m_{\mu^{+} \mu^{-}}-M_{Z}\right|$ and $\left|m_{\bar{\nu} \nu}-M_{Z}\right|>10 \mathrm{GeV}$ \\
Mass window & $\left|m_{\bar{\nu} \nu}-M_{Z^{\prime}}\right|<10 \mathrm{GeV}$ \\
\hline Number of muons & $N_{\mu}=2($ zero net charge $)$ \\
Number of taus & $N_{\tau_{\mathrm{h}}} \geq 1$ \\
$Z$ veto & $\left|m_{\mu^{+} \mu^{-}}-M_{Z}\right|$ and $\left|m_{\tau^{+} \tau^{-}}-M_{Z}\right|>10 \mathrm{GeV}$ \\
Mass window & $\left|m_{\mu^{+} \mu^{-}}-M_{Z^{\prime}}\right|$ or $\left|m_{\tau^{+} \tau^{-}}-M_{Z^{\prime}}\right|<10 \mathrm{GeV}$ \\
\hline
\end{tabular}

Table 3. From top to bottom, cuts imposed on the samples $4 \mu, 2 \mu 2 \nu_{\mu, \tau}$ with $Z^{\prime}$ decaying into muons, $2 \mu 2 \nu_{\mu, \tau}$ with $Z^{\prime}$ decaying into neutrinos and $2 \mu 2 \tau$ with $Z^{\prime}$ decaying into both muons and taus, respectively. $m_{\bar{\nu} \nu}$ and $m_{\tau^{+} \tau^{-}}$stand for the invariant mass reconstructed from the two observed muons and the initial total momentum, $\sqrt{\left(P-p_{\mu^{+}}-p_{\mu^{-}}\right)^{2}}$.
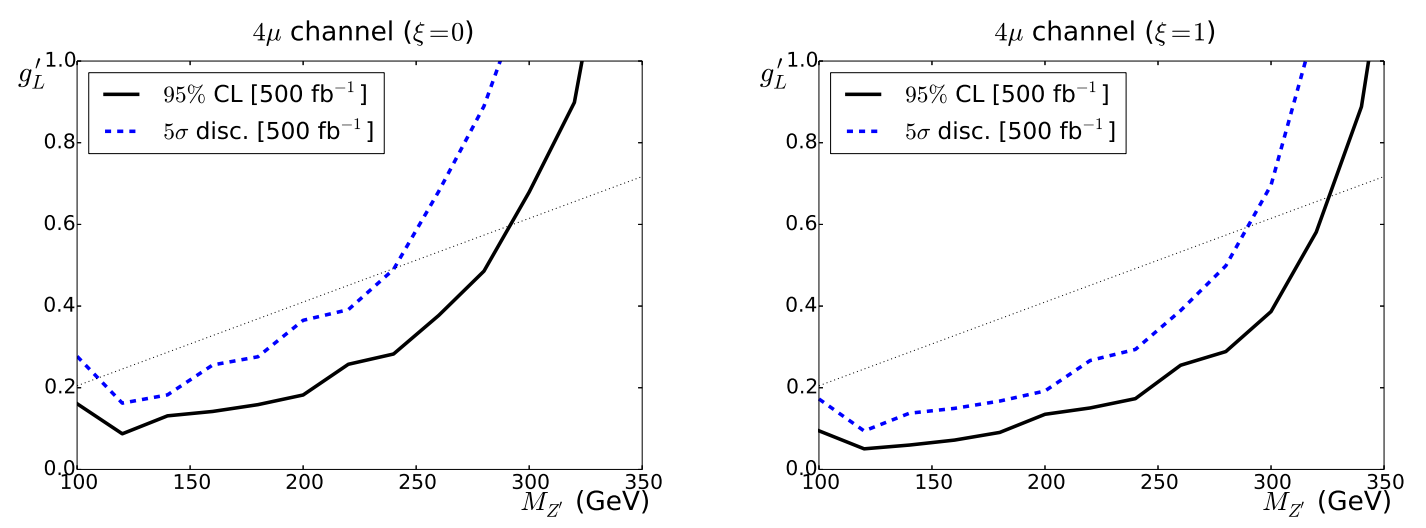

Figure 7. Discovery and exclusion limits for a leptophilic $Z^{\prime}$ as a function of its mass and its coupling to LH muons for $\xi=0$ (left) and $\xi=1$ (right) using only $4 \mu$ events at the ILC. We assume that no other $Z^{\prime}$ decay channel is open (in particular, that $Z^{\prime}$ does not decay into taus). The bound from neutrino trident production (straight line) is also shown for comparison. 


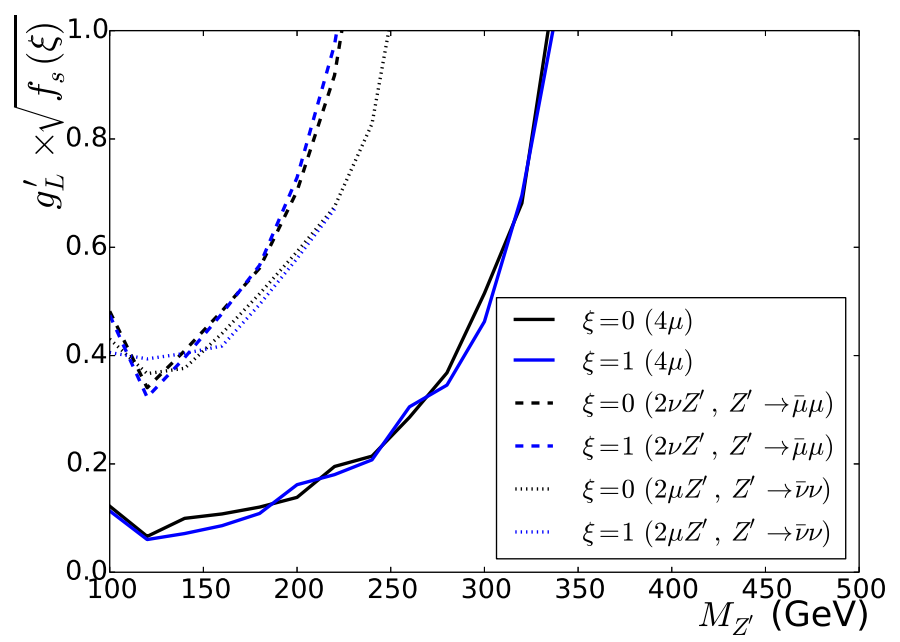

Figure 8. $95 \%$ C.L. exclusion limits for a leptophilic $Z^{\prime}$ as a function of its mass and its coupling to LH muons for two values of its coupling to their RH counterparts at the ILC, corrected for the new vector boson coupling dependence in eqs. (3.1): $f_{4 \mu}(\xi)=\left(1.15+\xi^{2}\right) \frac{1+\xi^{2}}{2+\xi^{2}}, f_{2 \mu}(\xi)=\frac{1+\xi^{2}}{2+\xi^{2}}$ for $Z^{\prime} \rightarrow \mu^{+} \mu^{-}$and $f_{2 \mu}(\xi)=\frac{1.15+\xi^{2}}{2+\xi^{2}}$ for $Z^{\prime} \rightarrow \bar{\nu} \nu$. We assume that the new gauge boson does not couple (decay) to tau leptons.
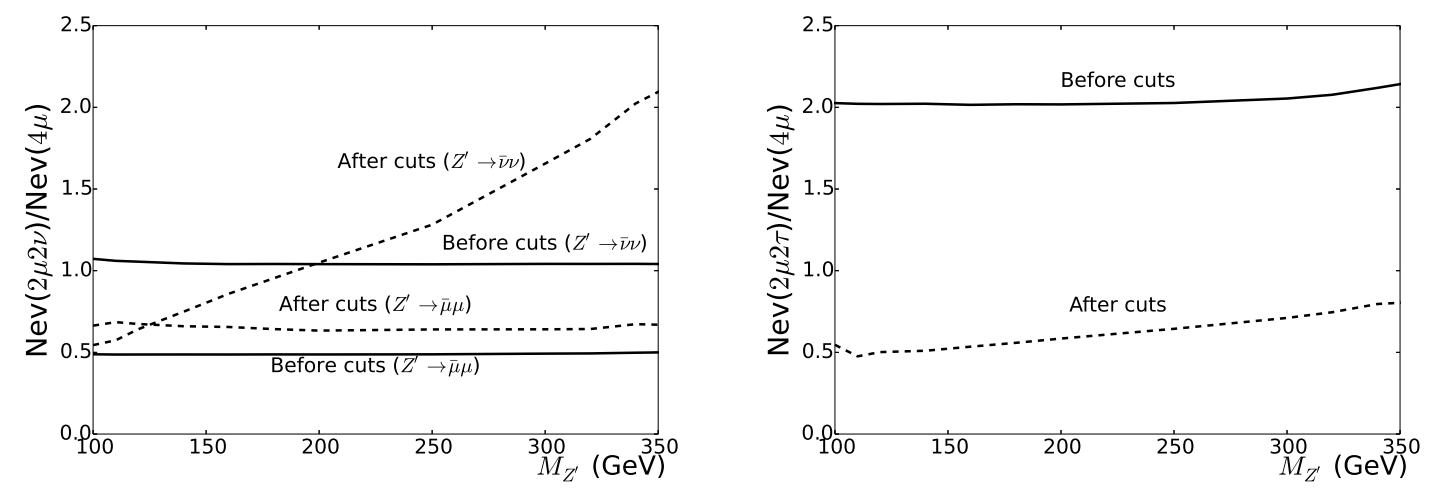

Figure 9. (Left) Ratio of $2 \mu$ plus missing energy to $4 \mu$ events as a function of the $Z_{\mu-\tau}^{\prime}$ mass for the anomaly-free model gauging $\mathrm{L}_{\mu}-\mathrm{L}_{\tau}$ at the ILC, distinguishing both vector boson decay modes $Z_{\mu-\tau}^{\prime} \rightarrow \mu^{+} \mu^{-}$and $\bar{\nu} \nu$. (Right) The same but for the ratio of $2 \mu 2 \tau$ to $4 \mu$ events.

couplings we must also confront the $2 \mu 2 \tau$ sample. Hence, in figure 9 we plot for the $\mathrm{L}_{\mu}-\mathrm{L}_{\tau}$ model the ratios of $2 \mu$ plus missing energy to $4 \mu$ events (left), distinguishing both $Z_{\mu-\tau}^{\prime}$ decays to $\mu^{+} \mu^{-}$and $\bar{\nu} \nu$, and of $2 \mu 2 \tau$ to $4 \mu$ events (right) as a function of the $Z_{\mu-\tau}^{\prime}$ mass. As can be observed, the curves before selection cuts closely follow the proportionality relations in eq. (3.2). What is not the case after selection cuts mainly due to the large sensitivity of some of the samples to the different cuts, especially to the fixed Mass window bin.

Finally, the total $Z^{\prime}$ width can be eventually measured at the ILC for a large enough statistics, but in this case the accessible $Z^{\prime}$ masses are lower than at the LHC (and its 


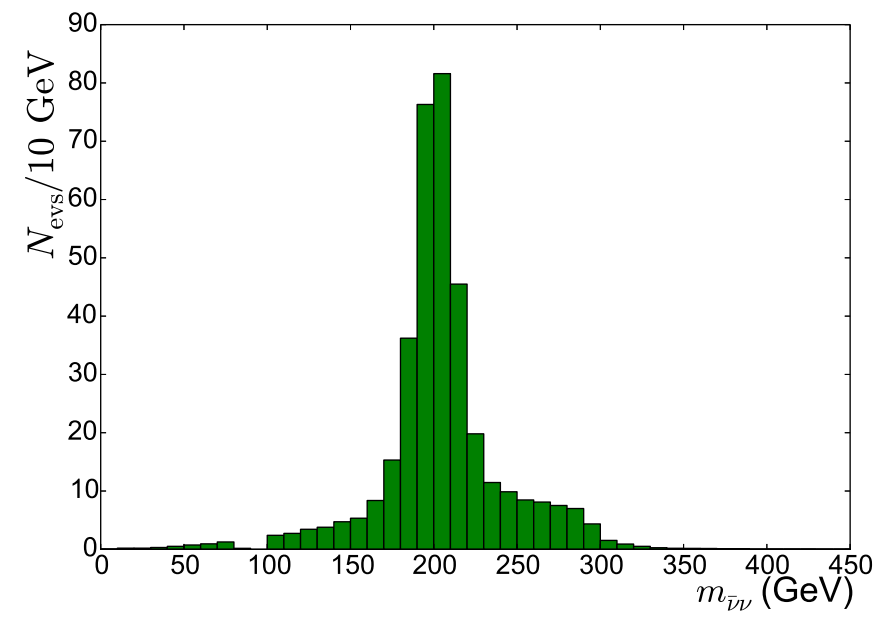

Figure 10. $Z_{\mu-\tau}^{\prime} \rightarrow \bar{\nu} \nu$ mass reconstruction in the $2 \mu 2 \nu$ channel for $M_{Z^{\prime}}=200 \mathrm{GeV}$ and $g_{\mathrm{L}}^{\prime}=1$.

alignment with the emitting lepton is less pronounced), as there are lower the muon momenta and then smaller the uncertainty in their determination. As already stated, the $Z^{\prime}$ width can be measured in two ways depending on whether we reconstruct the vector boson mass with two muons or two neutrinos, being the second one, $M_{Z^{\prime}}^{2}=\left(P-p_{\mu^{+}}-p_{\mu^{-}}\right)^{2}$, a priori more precise. In figure 10 we show the observed width $\sim 30 \mathrm{GeV}$ for a $Z^{\prime}$ of $200 \mathrm{GeV}$ and a total width of $16 \mathrm{GeV}$, assuming that the detector performance is the same as for the LHC, although it is aimed to be better.

In summary, once measured the total $Z^{\prime}$ width, and determined the muon couplings at the LHC, $g_{\mathrm{L}}^{\prime \tau}$ and $\xi_{\tau}$ can be measured at the ILC for low $Z^{\prime}$ masses, but most probably with a large statistical error. We have also investigated the possibility of improving the analysis of the $2 \mu 2 \tau$ sample by requiring not one (see the selection cuts in table 3 ) but both tau leptons to decay hadronically. However, although similar, the derived limits are less stringent and the tau coupling determination less precise for the heavier part of the spectrum due to the extra branching ratio suppression.

\section{Large collider bounds on four-lepton invariant operators}

The large collider limits on an extra vector boson only coupling to muons and taus, which we derived in previous sections, were obtained assuming a relatively narrow resonance and hence, an event excess around the vector boson mass. The non-observation of such an excess not only for any lepton-pair invariant mass but for the appropriate integrated region of fourlepton events can be also used to bound the size of the tail of new leptophilic interactions. In general these can be parametrized by the corresponding four-lepton operators, which contribute to four-lepton samples through the diagram(s) in figure 11. The cuts in this case, however, are different, as there are the bounds and the physical interpretation.

Let us first study the case of a $Z_{\mu-\tau}^{\prime}$ somewhat heavier than the LHC reach for illustration. The integration of this gauge boson out generates the four-lepton effective 


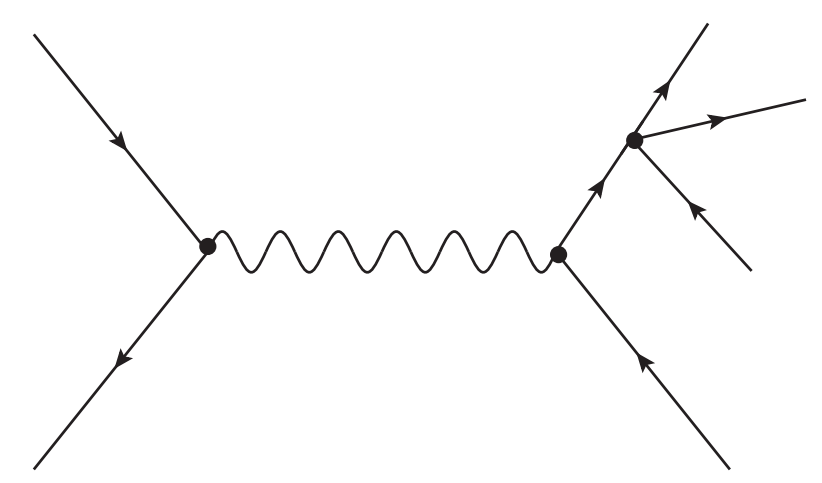

Figure 11. Leading contribution of four-lepton interactions not coupling to electrons at the LHC and ILC.

\begin{tabular}{|lll|}
\hline Scalar sum & $S_{\mathrm{T}}=\sum_{i=1}^{3}\left|p_{\mathrm{T}}^{\mu_{i}}\right|+E_{\mathrm{T}}>600 \mathrm{GeV}$, & $3 \mu$ plus missing energy channel \\
\hline Scalar sum & $S_{\mathrm{T}}=\sum_{i=1}^{4}\left|p_{\mathrm{T}}^{\mu_{i}}\right|>400 \mathrm{GeV}$, & $4 \mu$ channel \\
\hline
\end{tabular}

Table 4. Further cut which replaces the last cut (Mass window) in tables 1 and 2 for the $3 \mu$ plus missing energy and $4 \mu$ channels, respectively, in the absence of light resonances.

Lagrangian [16]:

$$
\begin{aligned}
\mathcal{L}_{\text {eff }}=-\frac{g_{\mathrm{L}}^{\prime 2}}{2 M_{Z_{\mu-\tau}^{\prime}}^{2}} & {\left[\left(\overline{L_{\mathrm{L} \mu}} \gamma_{\alpha} L_{\mathrm{L} \mu}\right)\left(\overline{L_{\mathrm{L} \mu}} \gamma^{\alpha} L_{\mathrm{L} \mu}\right)-2\left(\overline{L_{\mathrm{L} \mu}} \gamma_{\alpha} L_{\mathrm{L} \mu}\right)\left(\overline{L_{\mathrm{L} \tau}} \gamma^{\alpha} L_{\mathrm{L} \tau}\right)\right.} \\
& +\left(\overline{L_{\mathrm{L} \tau}} \gamma_{\alpha} L_{\mathrm{L} \tau}\right)\left(\overline{L_{\mathrm{L} \tau}} \gamma^{\alpha} L_{\mathrm{L} \tau}\right)+\left(\overline{\mu_{\mathrm{R}}} \gamma_{\alpha} \mu_{\mathrm{R}}\right)\left(\overline{\mu_{\mathrm{R}}} \gamma^{\alpha} \mu_{\mathrm{R}}\right) \\
& -2\left(\overline{\mu_{\mathrm{R}}} \gamma_{\alpha} \mu_{\mathrm{R}}\right)\left(\overline{\tau_{\mathrm{R}}} \gamma^{\alpha} \tau_{\mathrm{R}}\right)+\left(\overline{\tau_{\mathrm{R}}} \gamma_{\alpha} \tau_{\mathrm{R}}\right)\left(\overline{\tau_{\mathrm{R}}} \gamma^{\alpha} \tau_{\mathrm{R}}\right) \\
& -4\left(\overline{L_{\mathrm{L} \mu}} \mu_{\mathrm{R}}\right)\left(\overline{\mu_{\mathrm{R}}} L_{\mathrm{L} \mu}\right)+4\left(\overline{L_{\mathrm{L} \mu}} \tau_{\mathrm{R}}\right)\left(\overline{\tau_{\mathrm{R}}} L_{\mathrm{L} \mu}\right) \\
& \left.+4\left(\overline{L_{\mathrm{L} \tau}} \mu_{\mathrm{R}}\right)\left(\overline{\mu_{\mathrm{R}}} L_{\mathrm{L} \tau}\right)-4\left(\overline{L_{\mathrm{L} \tau}} \tau_{\mathrm{R}}\right)\left(\overline{\tau_{\mathrm{R}}} L_{\mathrm{L} \tau}\right)\right]
\end{aligned}
$$

which describes its low energy effects, and eventually the departure from the SM predictions for $4 \mu$ and $3 \mu$ plus missing energy distributions at the LHC. In order to confront this particular SM extension with an eventual excess or deficit of events in these samples, we have generated events as in previous sections but implementing the effective Lagrangian in eq. (4.1) in the UFO format [49]. We have then performed the corresponding analysis using also MADANALYSIS 5 [61, 62]. The cuts are the same as the ones in tables 1 and 2 except for the last one (Mass window) which is replaced by the corresponding cut in table 4 . This does not require the vector boson reconstruction but integrates the events on the tail of the kinematical distribution of the scalar transverse energy sum $S_{\mathrm{T}}$.

Assuming that no departure from the SM predictions is observed, we obtain an upper bound on the effective Lagrangian coefficient $x=\frac{g_{\mathrm{L}}^{\prime 2}}{2 M_{Z_{\mu-\tau}^{\prime}}^{2}}<3.8 \mathrm{TeV}^{-2}$ at $95 \%$ C.L., where we have closely followed the approach in ref. [19] to parametrize the effect of the four-lepton operators. This can be formally expressed as a limit on $M_{Z_{\mu-\tau}^{\prime}}>0.36 g_{\mathrm{L}}^{\prime} \mathrm{TeV}$, too. But this region of parameter space is mainly excluded by neutrino trident production, and by 


\begin{tabular}{|c|c|}
\hline$L L$ and $l l$ operators & $L l$ operators \\
\hline$\left(\mathcal{O}_{L L}^{(1)}\right)_{\mu \mu \mu \mu}=\frac{1}{2}\left(\overline{L_{\mathrm{L} \mu}} \gamma_{\alpha} L_{\mathrm{L} \mu}\right)\left(\overline{L_{\mathrm{L} \mu}} \gamma^{\alpha} L_{\mathrm{L} \mu}\right)$ & $\left(\mathcal{O}_{L l}\right)_{\mu \mu \mu \mu}=\left(\overline{L_{\mathrm{L} \mu}} \mu_{\mathrm{R}}\right)\left(\overline{\mu_{\mathrm{R}}} L_{\mathrm{L} \mu}\right)$ \\
$\left(\mathcal{O}_{L L}^{(1)}\right)_{\mu \mu \tau \tau}=\frac{1}{2}\left(\overline{L_{\mathrm{L} \mu}} \gamma_{\alpha} L_{\mathrm{L} \mu}\right)\left(\overline{L_{\mathrm{L} \tau}} \gamma^{\alpha} L_{\mathrm{L} \tau}\right)$ & $\left(\mathcal{O}_{L l}\right)_{\mu \mu \tau \tau}=\left(\overline{L_{\mathrm{L} \mu}} \mu_{\mathrm{R}}\right)\left(\overline{\tau_{\mathrm{R}}} L_{\mathrm{L} \tau}\right)$ \\
$\left(\mathcal{O}_{L L}^{(1)}\right)_{\mu \tau \mu \tau}=\frac{1}{2}\left(\overline{\bar{L}_{\mathrm{L} \mu}} \gamma_{\alpha} L_{\mathrm{L} \tau}\right)\left(\overline{L_{\mathrm{L} \mu}} \gamma^{\alpha} L_{\mathrm{L} \tau}\right)$ & $\left(\mathcal{O}_{L l}\right)_{\mu \tau \mu \tau}=\left(\overline{L_{\mathrm{L} \mu}} \tau_{\mathrm{R}}\right)\left(\overline{\mu_{\mathrm{R}}} L_{\mathrm{L} \tau}\right)$ \\
$\left(\mathcal{O}_{L L}^{(1)}\right)_{\tau \tau \tau \tau}=\frac{1}{2}\left(\overline{L_{\mathrm{L} \tau}} \gamma_{\alpha} L_{\mathrm{L} \tau}\right)\left(\overline{L_{\mathrm{L} \tau}} \gamma^{\alpha} L_{\mathrm{L} \tau}\right)$ & $\left(\mathcal{O}_{L l}\right)_{\mu \tau \tau \mu}=\left(\overline{L_{\mathrm{L} \mu}} \tau_{\mathrm{R}}\right)\left(\overline{\tau_{\mathrm{R}}} L_{\mathrm{L} \mu}\right)$ \\
$\left(\mathcal{O}_{l l}\right)_{\mu \mu \mu \mu}=\frac{1}{2}\left(\overline{\mu_{\mathrm{R}}} \gamma_{\alpha} \mu_{\mathrm{R}}\right)\left(\overline{\mu_{\mathrm{R}}} \gamma^{\alpha} \mu_{\mathrm{R}}\right)$ & $\left(\mathcal{O}_{L l}\right)_{\tau \mu \mu \tau}=\left(\overline{L_{\mathrm{L} \tau}} \mu_{\mathrm{R}}\right)\left(\overline{\mu_{\mathrm{R}}} L_{\mathrm{L} \tau}\right)$ \\
$\left(\mathcal{O}_{l l}\right)_{\mu \mu \tau \tau}=\frac{1}{2}\left(\overline{\mu_{\mathrm{R}}} \gamma_{\alpha} \mu_{\mathrm{R}}\right)\left(\overline{\tau_{\mathrm{R}}} \gamma^{\alpha} \tau_{\mathrm{R}}\right)$ & $\left(\mathcal{O}_{L l}\right)_{\tau \tau \tau \tau}=\left(\overline{L_{\mathrm{L} \tau}} \tau_{\mathrm{R}}\right)\left(\overline{\tau_{\mathrm{R}}} L_{\mathrm{L} \tau}\right)$ \\
$\left(\mathcal{O}_{l l}\right)_{\mu \tau \mu \tau}=\frac{1}{2}\left(\overline{\mu_{\mathrm{R}}} \gamma_{\alpha} \tau_{\mathrm{R}}\right)\left(\overline{\mu_{\mathrm{R}}} \gamma^{\alpha} \tau_{\mathrm{R}}\right)$ & \\
$\left(\mathcal{O}_{l l}\right)_{\tau \tau \tau \tau}=\frac{1}{2}\left(\overline{\tau_{\mathrm{R}}} \gamma_{\alpha} \tau_{\mathrm{R}}\right)\left(\overline{\tau_{\mathrm{R}}} \gamma^{\alpha} \tau_{\mathrm{R}}\right)$ & \\
\hline
\end{tabular}

Table 5. Independent four-lepton (gauge invariant) operators $\mathcal{O}_{4 l}$ involving muons and taus only. We assume flavor-parity conservation and omit hermitian conjugated partners.

the analysis presented in section 2 if no weakly coupled $\left(g_{\mathrm{L}}^{\prime}<\sqrt{4 \pi}\right)$ resonance $\left(Z_{\mu-\tau}^{\prime}\right)$ is found at the LHC (see figure 3 and footnote 10). Note that, on the other hand, in this particular case the limit on $x$ can be neither translated into a bound on $M_{Z_{\mu-\tau}^{\prime}}$ using its explicit dependence and hence, can not be interpreted as the limit on the effective coupling of a heavy resonance banished to a higher energy scale. This is always the case when the allowed values of $g_{\mathrm{L}}^{\prime}$ exceed $\sqrt{4 \pi}$ for masses not already excluded by the search of the corresponding weakly coupled resonances (at the LHC in our case).

Although in general this will be also the case for the LHC and ILC limits derived for any arbitrary combination of four-lepton operators involving muons and taus only, we can perform the same analysis as for $\mathcal{L}_{\text {eff }}$ in eq. (4.1) and obtain bounds on the corresponding effective Lagrangian coefficients. With this purpose in the following we define a basis of such four-lepton Lorentz and gauge invariant operators preserving flavor parity, and derive limits for the most favorable cases. Thus, in table 5 we list such an operator basis, up to hermitian conjugation. That this set is complete can be shown using the Fierz relation

$$
\left(\mathcal{O}_{L L}^{(3)}\right)_{i j k l}=2\left(\mathcal{O}_{L L}^{(1)}\right)_{i l k j}-\left(\mathcal{O}_{L L}^{(1)}\right)_{i j k l}
$$

to reduce the four combinations of four SM lepton multiplets which are Lorentz and gauge invariant:

$$
\begin{aligned}
\left(\mathcal{O}_{L L}^{(1)}\right)_{i j k l} & =\frac{1}{2}\left(\overline{L_{\mathrm{L} i}} \gamma_{\alpha} L_{\mathrm{L} j}\right)\left(\overline{L_{\mathrm{L} k}} \gamma^{\alpha} L_{\mathrm{L} l}\right), \\
\left(\mathcal{O}_{L L}^{(3)}\right)_{i j k l} & =\frac{1}{2}\left(\overline{L_{\mathrm{L} i}} \gamma_{\alpha} \sigma_{a} L_{\mathrm{L} j}\right)\left(\overline{L_{\mathrm{L} k}} \gamma^{\alpha} \sigma_{a} L_{\mathrm{L} l}\right), \\
\left(\mathcal{O}_{l l}\right)_{i j k l} & =\left(\overline{\overline{\mathrm{R}}_{\mathrm{i}}} \gamma_{\alpha} l_{\mathrm{R} j}\right)\left(\overline{\overline{\mathrm{R}}_{\mathrm{R}}} \gamma^{\alpha} l_{\mathrm{R} l}\right), \\
\left(\mathcal{O}_{L l}\right)_{i j k l} & =\left(\overline{L_{\mathrm{L} i}} l_{\mathrm{R} j}\right)\left(\overline{l_{\mathrm{R} k}} L_{\mathrm{L} l}\right),
\end{aligned}
$$

to three. These operators are also invariant under the exchange of the pairs of flavor indices $(i j) \leftrightarrow(k l)$ and under the permutation of the two flavor indices $i \leftrightarrow k$ and therefore, under the permutation of the other two flavor indices $j \leftrightarrow l$. What reduces the independent four-lepton operators $\mathcal{O}_{4 l}$ to the set in table 5 if we also require flavor-parity conservation. 
Lepton number conservation follows from gauge invariance at this order. Then, any NP coupling to muons and taus and heavy enough to evade direct observation at the LHC can be parametrized by a combination of the operators in this table. We estimate the corresponding LHC and ILC reach assuming that only one of them has a non-vanishing coefficient at a time. The most stringent bounds are obtained for the four-muon operators $\left(\mathcal{O}_{L L}^{(1)}\right)_{\mu \mu \mu \mu},\left(\mathcal{O}_{l l}\right)_{\mu \mu \mu \mu}$ and $\left(\mathcal{O}_{L l}\right)_{\mu \mu \mu \mu}$. Using the same generation procedure as for $\mathcal{L}_{\text {eff }}$ in eq. (4.1) and applying the same cuts as in table 4 (and in tables 1 and 2 except for the Mass window cut) to the $3 \mu$ plus missing energy and $4 \mu$ samples, respectively, we obtain the $95 \%$ C.L. limits at the LHC: ${ }^{13}$

$$
\begin{aligned}
-10 \mathrm{TeV}^{-2} & \leq x^{L L} \leq 8.9 \mathrm{TeV}^{-2}, \\
-10.8 \mathrm{TeV}^{-2} & \leq x^{l l} \leq 10.4 \mathrm{TeV}^{-2}, \\
-11.2 \mathrm{TeV}^{-2} \leq x^{L l} & \leq 12.2 \mathrm{TeV}^{-2},
\end{aligned}
$$

and at the ILC:

$$
\begin{aligned}
& -38 \mathrm{TeV}^{-2} \leq x^{L L} \leq 25 \mathrm{TeV}^{-2} \\
& -39 \mathrm{TeV}^{-2} \leq x^{l l} \leq 27 \mathrm{TeV}^{-2} \\
& -24 \mathrm{TeV}^{-2} \leq x^{L l} \leq 31 \mathrm{TeV}^{-2}
\end{aligned}
$$

Values which are similar to those derived for the heavy $Z_{\mu-\tau}^{\prime}$ effective Lagrangian in eq. (4.1), as similar are the applicable comments.

If the leptophilic effective operators are allowed to involve electrons, too, there are 19 more independent four-lepton operators and an estimate of present bounds is reviewed in the Introduction.

\section{Conclusions}

The outstanding performance of the LHC experiments makes them not only discovery but also precision devices. With this in mind it is natural to question whether the ILC is really a complementary machine, especially if no signal of NP is established at the LHC. At any rate, the energy frontier is the domain of hadron machines, as are the strong interactions, and at the end of the day even the EW gauge interactions, too. In general only the eventually huge backgrounds set the limits of the LHC potential. Hence, an obvious question which we have addressed in this paper is if NP only coupling to leptons and naturally accessible to the ILC is constrained at all by a large hadron collider. The only lowest order interaction fulfilling these conditions is a new leptophilic neutral vector boson.

We have explored this SM addition allowing for general couplings to muons and taus (and to their neutral counterparts). Couplings to electrons can be neglected because the corresponding LEP limits make the LHC insensitive to them, as LFV bounds make the LHC insensitive to leptophilic off-diagonal couplings. As a matter of fact, LHC experiments are only sensitive to the $Z^{\prime}$ couplings to muons because couplings to taus can not be

\footnotetext{
${ }^{13}$ The effective Lagrangian is defined as $x^{i} \mathcal{O}_{i}$, with no additional global sign.
} 
efficiently measured due to their larger backgrounds. The LHC reach for a leptophilic $Z^{\prime}$ coupling to muons can be up to $\sim 1 \mathrm{TeV}$ for coupling constants of order one. Moreover, the comparison of the $3 \mu$ plus missing energy and $4 \mu$ final states allows to determine the ratio of the $\mathrm{RH}$ singlet to the $\mathrm{LH}$ doublet muon couplings. In order to also determine the global normalization, the $Z^{\prime}$ total width must be independently measured, which is not easy at the LHC (see figure 6). The corresponding determination of these couplings at the ILC relies on the $4 \mu, 2 \mu$ plus missing energy and $2 \mu 2 \tau$ samples (in the second and third sets two subsamples can be distinguished depending on whether the $Z^{\prime}$ decays into $\mu^{+} \mu^{-}$ or $\bar{\nu} \nu$ or into $\mu^{+} \mu^{-}$or $\tau^{+} \tau^{-}$, respectively), since the initial state is neutral and no $W$ can be exchanged in the $s$-channel. The bounds which may be eventually derived are similar to the LHC ones, but only for light enough vector boson masses $(<250 \mathrm{GeV})$. In contrast, the $Z^{\prime}$ couplings to taus can be only determined at the ILC in either case. However, the sensitivity of the ILC is very much dependent not only on how low the new vector boson mass is but also on the relative size of $g_{\mathrm{L}, \mathrm{R}}^{\prime \mu}$ and $g_{\mathrm{L}, \mathrm{R}}^{\prime \tau}$, because the former must be sizable for reconstructing the $Z^{\prime}$ and the latter for producing a large enough signal to measure the tau couplings. This appreciably reduces the accessible parameter space at the ILC.

In summary, the only departure from the SM eventually observable a priori at the LHC, if a new leptophilic vector boson exists, is a moderate excess of events in the $3 \mu$ plus missing energy and $4 \mu$ samples with a $\mu^{+} \mu^{-}$pair peaking around the $Z^{\prime}$ mass. The typical cross-section after preselection cuts being of few $\mathrm{fb}$. On the other hand, the ILC can only confirm the observation of such a vector boson. Although for a rather light $Z^{\prime}$ also coupling sizably to taus, an analysis of the $2 \mu$ plus missing energy and $2 \mu 2 \tau$ samples could also allow for the measurement of the $Z^{\prime}$ couplings to taus. If this leptophilic vector boson would also couple to electrons, the leptonic processes $e^{+} e^{-} \rightarrow \mu^{+} \mu^{-}, \tau^{+} \tau^{-}$would offer the best place to look for such a new interaction $[17,18]$.

We have also discussed the limit of a $Z^{\prime}$ with a mass beyond the LHC reach, that can be parametrized by the corresponding four-lepton operators. Limits on the independent invariant (dimension 6) operators involving four SM lepton multiplets can be estimated in a similar way, but the corresponding bounds on their coefficients, $\sim 10 \mathrm{TeV}^{-2}$, are too weak to allow for a weakly coupled resonance interpretation.

The fast simulation analyses have been performed using MADGRAPH 5 [52]. The UFO model can be found in http://cafpe.ugr.es/index.php/pages/other/software in the package Leptophilic_UFO.tar.gz.

\section{Acknowledgments}

We thank J . Alcaraz, S. Bethke and T. Golling for useful discussions. MC would also like to thank the Institute for Theoretical Physics at ETH Zürich for hospitality during the completion of this project. This work has been supported in part by the European Commission through the contract PITN-GA-2012-316704 (HIGGSTOOLS), by the Ministry of Economy and Competitiveness (MINECO), under grant numbers FPA2010-17915 and FPA2013-47836-C3-1/2-P, and by the Junta de Andalucía grants FQM 101 and FQM 6552. M.C. was supported by the MINECO under the FPU program. 
Open Access. This article is distributed under the terms of the Creative Commons Attribution License (CC-BY 4.0), which permits any use, distribution and reproduction in any medium, provided the original author(s) and source are credited.

\section{References}

[1] F. Englert and R. Brout, Broken symmetry and the mass of gauge vector mesons, Phys. Rev. Lett. 13 (1964) 321 [inSPIRE].

[2] P.W. Higgs, Broken symmetries and the masses of gauge bosons, Phys. Rev. Lett. 13 (1964) 508 [INSPIRE].

[3] ATLAS collaboration, Observation of a new particle in the search for the standard model Higgs boson with the ATLAS detector at the LHC, Phys. Lett. B 716 (2012) 1 [arXiv: 1207.7214] [INSPIRE].

[4] CMS collaboration, Observation of a new boson at a mass of $125 \mathrm{GeV}$ with the CMS experiment at the LHC, Phys. Lett. B $\mathbf{7 1 6}$ (2012) 30 [arXiv:1207.7235] [INSPIRE].

[5] CMS collaboration, Search for narrow resonances using the dijet mass spectrum in pp collisions at $\sqrt{s}=8 \mathrm{TeV}$, Phys. Rev. D 87 (2013) 114015 [arXiv:1302.4794] [INSPIRE].

[6] ATLAS collaboration, Search for new phenomena in the dijet mass distribution using pp collision data at $\sqrt{s}=8 \mathrm{TeV}$ with the ATLAS detector, arXiv:1407.1376 [INSPIRE].

[7] CMS collaboration, Search for massive resonances in dijet systems containing jets tagged as $W$ or $Z$ boson decays in pp collisions at $\sqrt{s}=8 \mathrm{TeV}$, JHEP 08 (2014) 173 [arXiv: 1405.1994] [INSPIRE].

[8] ATLAS collaboration, Search for pair and single production of new heavy quarks that decay to a $Z$ boson and a third-generation quark in pp collisions at $\sqrt{s}=8$ TeV with the ATLAS detector, JHEP 11 (2014) 104 [arXiv:1409.5500] [INSPIRE].

[9] CMS collaboration, Search for top-quark partners with charge $5 / 3$ in the same-sign dilepton final state, Phys. Rev. Lett. 112 (2014) 171801 [arXiv:1312.2391] [INSPIRE].

[10] CMS collaboration, Inclusive search for a vector-like $T$ quark with charge $2 / 3$ in pp collisions at $\sqrt{s}=8 \mathrm{TeV}$, Phys. Lett. B $\mathbf{7 2 9}$ (2014) 149 [arXiv:1311.7667] [inSPIRE].

[11] CMS collaboration, Search for gluino mediated bottom- and top-squark production in multijet final states in pp collisions at 8 TeV, Phys. Lett. B 725 (2013) 243 [arXiv:1305.2390] [INSPIRE].

[12] ATLAS collaboration, Search for squarks and gluinos with the ATLAS detector in final states with jets and missing transverse momentum using $\sqrt{s}=8$ TeV proton-proton collision data, JHEP 09 (2014) 176 [arXiv:1405.7875] [INSPIRE].

[13] CMS collaboration, Search for new physics in the multijet and missing transverse momentum final state in proton-proton collisions at $\sqrt{s}=8 \mathrm{TeV}$, JHEP 06 (2014) 055 [arXiv: 1402.4770] [INSPIRE].

[14] CMS collaboration, Search for heavy narrow dilepton resonances in pp collisions at $\sqrt{s}=7 \mathrm{TeV}$ and $\sqrt{s}=8 \mathrm{TeV}$, Phys. Lett. B 720 (2013) 63 [arXiv: 1212.6175] [InSPIRE].

[15] ATLAS collaboration, Search for high-mass dilepton resonances in pp collisions at $\sqrt{s}=8 \mathrm{TeV}$ with the ATLAS detector, Phys. Rev. D 90 (2014) 052005 [arXiv:1405.4123] [INSPIRE]. 
[16] F. del Aguila, J. de Blas and M. Pérez-Victoria, Electroweak limits on general new vector bosons, JHEP 09 (2010) 033 [arXiv: 1005.3998] [INSPIRE].

[17] F. del Aguila and J. de Blas, Electroweak constraints on new physics, Fortsch. Phys. 59 (2011) 1036 [arXiv:1105.6103] [INSPIRE].

[18] J. de Blas, Electroweak limits on physics beyond the standard model, EPJ Web Conf. 60 (2013) 19008 [arXiv: 1307.6173] [INSPIRE].

[19] J. de Blas, M. Chala and J. Santiago, Global constraints on lepton-quark contact interactions, Phys. Rev. D 88 (2013) 095011 [arXiv:1307.5068] [INSPIRE].

[20] H. Baer et al., The International Linear Collider technical design report - volume 2: physics, arXiv:1306.6352 [INSPIRE].

[21] F. Del Aguila and M. Cvetič, Diagnostic power of future colliders for $Z^{\prime}$ couplings to quarks and leptons: $e^{+} e^{-}$versus pp colliders, Phys. Rev. D 50 (1994) 3158 [hep-ph/9312329] [INSPIRE].

[22] F. Del Aguila, M. Cvetič and P. Langacker, Reconstruction of the extended gauge structure from $Z^{\prime}$ observables at future colliders, Phys. Rev. D 52 (1995) 37 [hep-ph/9501390] [INSPIRE].

[23] A. Freitas et al., Exploring quantum physics at the ILC, arXiv:1307.3962 [INSPIRE].

[24] F. del Aguila, M. Chala, A. Santamaria and J. Wudka, Discriminating between lepton number violating scalars using events with four and three charged leptons at the LHC, Phys. Lett. B 725 (2013) 310 [arXiv: 1305.3904] [INSPIRE].

[25] F. del Aguila, M. Chala, A. Santamaria and J. Wudka, Distinguishing between lepton number violating scalars at the LHC, EPJ Web Conf. 60 (2013) 17002 [arXiv:1307.0510] [INSPIRE].

[26] F. del Águila and M. Chala, LHC bounds on lepton number violation mediated by doubly and singly-charged scalars, JHEP 03 (2014) 027 [arXiv:1311.1510] [INSPIRE].

[27] F. del Aguila, M. Chala, A. Santamaria and J. Wudka, Lepton number violation and scalar searches at the LHC, Acta Phys. Polon. B 44 (2013) 2139 [arXiv:1311.2950] [InSPIRE].

[28] B. Holdom, Two U(1)'s and $\epsilon$ charge shifts, Phys. Lett. B 166 (1986) 196 [INSPIRE].

[29] F. del Aguila, G.A. Blair, M. Daniel and G.G. Ross, Superstring inspired models, Nucl. Phys. B 272 (1986) 413 [INSPIRE].

[30] F. del Aguila, G.D. Coughlan and M. Quirós, Gauge coupling renormalization with several U(1) factors, Nucl. Phys. B 307 (1988) 633 [Erratum ibid. B 312 (1989) 751] [INSPIRE].

[31] C.D. Carone and H. Murayama, Realistic models with a light U(1) gauge boson coupled to baryon number, Phys. Rev. D 52 (1995) 484 [hep-ph/9501220] [INSPIRE].

[32] F. del Aguila, M. Masip and M. Pérez-Victoria, Physical parameters and renormalization of $\mathrm{U}(1)^{a} \times \mathrm{U}(1)^{b}$ models, Nucl. Phys. B 456 (1995) 531 [hep-ph/9507455] [INSPIRE].

[33] V.V. Andreev, P. Osland and A.A. Pankov, Precise determination of $Z-Z^{\prime}$ mixing at the CERN LHC, Phys. Rev. D 90 (2014) 055025 [arXiv:1406.6776] [INSPIRE].

[34] F. Aguila, M. Chala, J. Santiago and Y. Yamamoto, Four-lepton signals of leptophilic gauge interactions at large colliders, in preparation.

[35] R. Foot, X.G. He, H. Lew and R.R. Volkas, Model for a light $Z^{\prime}$ boson, Phys. Rev. D 50 (1994) 4571 [hep-ph/9401250] [INSPIRE]. 
[36] F. del Aguila, A. Carmona and J. Santiago, Neutrino masses from an $A_{4}$ symmetry in holographic composite Higgs models, JHEP 08 (2010) 127 [arXiv:1001.5151] [INSPIRE].

[37] F. del Aguila, A. Carmona and J. Santiago, Tau custodian searches at the LHC, Phys. Lett. B 695 (2011) 449 [arXiv:1007.4206] [InSPIRE].

[38] T. Han and B. Zhang, Signatures for Majorana neutrinos at hadron colliders, Phys. Rev. Lett. 97 (2006) 171804 [hep-ph/0604064] [INSPIRE].

[39] F. del Aguila, J.A. Aguilar-Saavedra and R. Pittau, Heavy neutrino signals at Large Hadron Colliders, JHEP 10 (2007) 047 [hep-ph/0703261] [INSPIRE].

[40] A. Atre, T. Han, S. Pascoli and B. Zhang, The search for heavy Majorana neutrinos, JHEP 05 (2009) 030 [arXiv:0901.3589] [INSPIRE].

[41] P.S.B. Dev, A. Pilaftsis and U.-K. Yang, New production mechanism for heavy neutrinos at the LHC, Phys. Rev. Lett. 112 (2014) 081801 [arXiv:1308.2209] [InSPIRE].

[42] ALEPH, DELPHI, L3, OPAL and LEP EleCtrowEAK collaborations, S. Schael et al., Electroweak measurements in electron-positron collisions at $W$-boson-pair energies at LEP, Phys. Rept. 532 (2013) 119 [arXiv:1302.3415] [INSPIRE].

[43] SINDRUM collaboration, U. Bellgardt et al., Search for the decay $\mu^{+} \rightarrow e^{+} e^{+} e^{-}$, Nucl. Phys. B 299 (1988) 1 [INSPIRE].

[44] K. Hayasaka et al., Search for lepton flavor violating $\tau$ decays into three leptons with 719 million produced $\tau^{+} \tau^{-}$pairs, Phys. Lett. B 687 (2010) 139 [arXiv:1001.3221] [INSPIRE].

[45] M. Cvetič and P. Langacker, $V^{\prime} Z$ and $V^{\prime} W$ production as tests of heavy gauge boson couplings at future hadron colliders, Phys. Rev. D 46 (1992) 4943 [Erratum ibid. D 48 (1993) 4484] [hep-ph/9207216] [INSPIRE].

[46] M. Cvetič, P. Langacker and B. Kayser, Determination of $g_{R} / g_{L}$ in left-right symmetric models at hadron colliders, Phys. Rev. Lett. 68 (1992) 2871 [INSPIRE].

[47] F. del Aguila, B. Alles, L. Ametller and A. Grau, $Z^{\prime}$ decays in four fermions, Phys. Rev. D 48 (1993) 425 [hep-ph/9206219] [INSPIRE].

[48] E. Fuchs, S. Thewes and G. Weiglein, Interference effects in BSM processes with a generalised narrow-width approximation, arXiv:1411.4652 [INSPIRE].

[49] C. Degrande et al., UFO - the Universal FeynRules Output, Comput. Phys. Commun. 183 (2012) 1201 [arXiv:1108.2040] [InSPIRE].

[50] N.D. Christensen and C. Duhr, FeynRules - Feynman rules made easy, Comput. Phys. Commun. 180 (2009) 1614 [arXiv:0806.4194] [InSPIRE].

[51] A. Alloul, N.D. Christensen, C. Degrande, C. Duhr and B. Fuks, FeynRules $2.0-a$ complete toolbox for tree-level phenomenology, Comput. Phys. Commun. 185 (2014) 2250 [arXiv:1310.1921] [INSPIRE].

[52] J. Alwall, M. Herquet, F. Maltoni, O. Mattelaer and T. Stelzer, MadGraph 5: going beyond, JHEP 06 (2011) 128 [arXiv:1106.0522] [INSPIRE].

[53] J. Heeck and W. Rodejohann, Gauged $L_{\mu}-L_{\tau}$ symmetry at the electroweak scale, Phys. Rev. D 84 (2011) 075007 [arXiv:1107.5238] [INSPIRE].

[54] K. Harigaya, T. Igari, M.M. Nojiri, M. Takeuchi and K. Tobe, Muon g-2 and LHC phenomenology in the $L_{\mu}-L_{\tau}$ gauge symmetric model, JHEP 03 (2014) 105 [arXiv:1311.0870] [INSPIRE]. 
[55] N.F. Bell, Y. Cai, R.K. Leane and A.D. Medina, Leptophilic dark matter with $Z^{\prime}$ interactions, Phys. Rev. D 90 (2014) 035027 [arXiv: 1407.3001] [InSPIRE].

[56] E. Ma, D.P. Roy and S. Roy, Gauged $L_{\mu}-L_{\tau}$ with large muon anomalous magnetic moment and the bimaximal mixing of neutrinos, Phys. Lett. B 525 (2002) 101 [hep-ph/0110146] [INSPIRE].

[57] W. Altmannshofer, S. Gori, M. Pospelov and I. Yavin, Quark flavor transitions in $L_{\mu}-L_{\tau}$ models, Phys. Rev. D 89 (2014) 095033 [arXiv: 1403.1269] [InSPIRE].

[58] W. Altmannshofer, S. Gori, M. Pospelov and I. Yavin, Neutrino trident production: a powerful probe of new physics with neutrino beams, Phys. Rev. Lett. 113 (2014) 091801 [arXiv: 1406.2332] [INSPIRE].

[59] T. Sjöstrand, S. Mrenna and P.Z. Skands, PYTHIA 6.4 physics and manual, JHEP 05 (2006) 026 [hep-ph/0603175] [INSPIRE].

[60] DELPHES 3 collaboration, J. de Favereau et al., DELPHES 3, a modular framework for fast simulation of a generic collider experiment, JHEP 02 (2014) 057 [arXiv:1307.6346] [INSPIRE].

[61] E. Conte, B. Fuks and G. Serret, MadAnalysis 5, a user-friendly framework for collider phenomenology, Comput. Phys. Commun. 184 (2013) 222 [arXiv:1206.1599] [INSPIRE].

[62] E. Conte, B. Dumont, B. Fuks and C. Wymant, Designing and recasting LHC analyses with MadAnalysis 5, Eur. Phys. J. C 74 (2014) 3103 [arXiv: 1405.3982] [INSPIRE].

[63] M. Cacciari, G.P. Salam and G. Soyez, FastJet user manual, Eur. Phys. J. C 72 (2012) 1896 [arXiv:1111.6097] [INSPIRE].

[64] ATLAS collaboration, Search for direct production of charginos and neutralinos in events with three leptons and missing transverse momentum in $21 \mathrm{fb}^{-1}$ of pp collisions at $\sqrt{s}=8 \mathrm{TeV}$ with the ATLAS detector, ATLAS-CONF-2013-035, CERN, Geneva Switzerland (2013).

[65] Particle Data Group collaboration, K.A. Olive et al., Review of particle physics, Chin. Phys. C 38 (2014) 090001 [inSPIRE].

[66] G. Cowan, K. Cranmer, E. Gross and O. Vitells, Asymptotic formulae for likelihood-based tests of new physics, Eur. Phys. J. C 71 (2011) 1554 [Erratum ibid. C 73 (2013) 2501] [arXiv: 1007.1727] [INSPIRE]. 